UNIVERSIDADE DE SÃO PAULO

FACULDADE DE FILOSOFIA, LETRAS E CIÊNCIAS HUMANAS

DEPARTAMENTO DE FILOSOFIA

PROGRAMA DE PÓS-GRADUAÇÃO EM FILOSOFIA

René Pierre Maximilian Eduard Mazak

\title{
Um Sistema de Gentzen para Cálculos com Identidade Parcial e Universos Abertos
}


UNIVERSIDADE DE SÃO PAULO

FACULDADE DE FILOSOFIA, LETRAS E CIÊNCIAS

HUMANAS

DEPARTAMENTO DE FILOSOFIA

PROGRAMA DE PÓS-GRADUAÇÃO EM FILOSOFIA

\section{Um Sistema de Gentzen para Cálculos com Identidade Parcial e Universos Abertos}

René Pierre Maximilian Eduard Mazak

Dissertação apresentada ao

Programa de Pós-Graduação em

Filosofia do Departamento de

Filosofia da Faculdade de

Filosofia, Letras e Ciências Humanas da Universidade de São Paulo, para a obtenção do título de Mestre em Filosofia.

Orientadora: Profa. Dra. Andréa Maria Altino de Campos Loparić 


\section{Resumo}

Os sistemas $\mathrm{Q}_{1}$ e $\mathrm{Q}_{2}$, desenvolvidos por Andréa Loparić, perfazem três principais modificações na semântica clássica: primeiramente, o universo do discurso pode não estar limitado aos objetos que pertencem ao domínio de uma dada estrutura; em segundo lugar, a relação de identidade é determinada como a diagonal desse domínio (assim, tal relação pode não ser aplicável a todas as coisas sobre as quais a linguagem possa falar); em terceiro lugar, o quantificador existencial, em $\mathrm{Q}_{1}$, bem como o universal, em $\mathrm{Q}_{2}$, podem alcançar valores que estejam fora do domínio da estrutura. Como consequência, embora definida classicamente, a negação apresenta alguns comportamentos não clássicos - a negação de um predicado numa fórmula atômica, por exemplo, pode caracterizar algo maior que, e não tão bem definido quanto, o complemento da extensão desse predicado relativamente ao domínio.

Posteriormente, o sistema $\mathrm{Q}_{1}$ foi estendido por Fernando Paulo Christe Adorno para incluir nomes e símbolos funcionais em sua linguagem. Adorno apresentou também: uma nova extensão desse sistema estendido, chamada $\mathrm{Q}_{1 \mathrm{p}}$, que inclui parâmetros entre seus símbolos individuais e é uma extensão conservativa de $\mathrm{Q}_{1}$; e um sistema de dedução natural, chamado $\mathrm{S}$, que foi demonstrado ser correto e completo relativamente a $\mathrm{Q}_{1 \mathrm{p}}$. Dois outros resultados foram também demonstrados: 1) para toda formula $\alpha$ da linguagem de $\mathrm{Q}_{1}, \alpha$ é válida em $\mathrm{Q}_{1}$ se e somente se $\alpha$ é um teorema de $\mathrm{S}$; e 2) para toda formula $\alpha$ em $\mathrm{Q}_{2}$, há uma formula $\alpha^{*}$ em $\mathrm{Q}_{1}$ tal que $\alpha$ é $\mathrm{Q}_{2}$-válida sse $\alpha^{*}$ é $\mathrm{Q}_{1}$-válida.

Em nosso trabalho, apresentamos um estudo detalhado dos sistemas $\mathrm{Q}_{1} \mathrm{e} \mathrm{Q}_{2}$, na primeira parte; na segunda parte, um novo sistema de Gentzen, chamado $\mathrm{QG}_{1}$, que trabalha com sequentes, e resulta de algumas modificações no sistema LK, de Gentzen. Por fim, na terceira parte, provamos que o sistema $\mathrm{QG}_{1}$ é equivalente ao sistema $\mathrm{S}$. 


\section{Abstract}

The systems $\mathrm{Q}_{1}$ and $\mathrm{Q}_{2}$, developed by Andréa Loparić, make up three main changes in classical semantics: first, the universe of discourse can be not limited by the objects that belongs to the domain of a given structure; second, the relation of identity is fixed as a diagonal of this domain (so, it may be not applicable to all things about what the language can speak); third, the existential quantifier in $\mathrm{Q}_{1}$, as well as the universal in $\mathrm{Q}_{2}$, may capture values out of the domain of the structure. As a consequence, although classically defined, the negation presents some non-classical behavior - a negated predicate in an atomic formula, for instance, may characterize something larger and not as well defined as the complement of the extension of this predicate relatively to the domain.

Further, the system $\mathrm{Q}_{1}$ was extended by Fernando Paulo Christe Adorno to include names and functional symbols in its language. Adorno has also presented: a new extension of this extended system, called $\mathrm{Q}_{1 \mathrm{p}}$, which includes parameters among its individual symbols and is a conservative extension of $\mathrm{Q}_{1}$; and a system of natural deduction, called $\mathrm{S}$, which was proved to be sound and complete relatively to $\mathrm{Q}_{1 \mathrm{p}}$. Two other results were also proved: 1) for every formula $\alpha$ of $\mathrm{Q}_{1}$ 's language, $\alpha$ is valid in $\mathrm{Q}_{1}$ if and only if $\alpha$ is a theorem of $\mathrm{S}$; and 2) for every formula $\alpha$ in $\mathrm{Q}_{2}$, there is a formula $\alpha^{*}$ in $\mathrm{Q}_{1}$ such that $\alpha$ is $\mathrm{Q}_{2}$-valid iff $\alpha^{*}$ is $\mathrm{Q}_{1}$-valid.

In our work, we present a detailed study of the systems $\mathrm{Q}_{1}$ and $\mathrm{Q}_{2}$, in first part; in second one, a new Gentzen-type system, called $\mathrm{QG}_{1}$, which works with sequents, and results of some changings in the Gentzen system LK. Finally, in third part, we prove that the system $\mathrm{QG}_{1}$ is equivalent to the system $\mathrm{S}$. 
Palavras-chave: Sistemas de Gentzen; Quantificadores Não Clássicos; Identidade Parcial; Universos Abertos; Negações e Predicações; Objetos e Ficções.

Keywords: Gentzen Systems; Non-Classical Quantifiers; Partial Identity; Open Universes; Negations and Predications; Objects and Fictions. 


\section{Sumário}

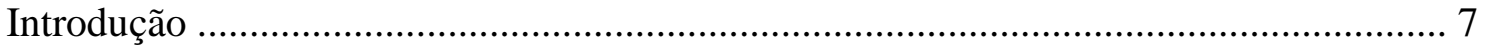

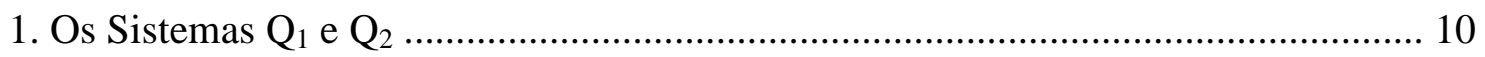

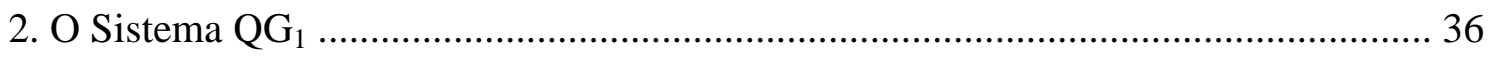

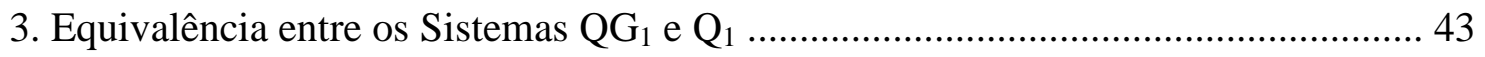

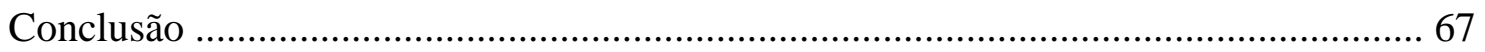

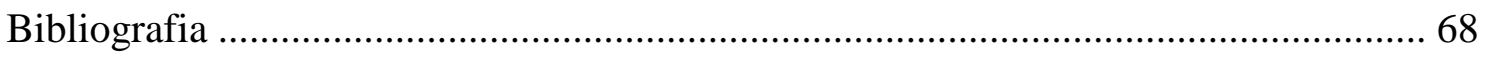




\section{Introdução}

A presente dissertação é o produto de meu interesse por dois conjuntos de sistemas lógicos: por um lado, os sistemas de Gentzen (em particular, os sistemas de dedução lógica, LJ e LK, que trabalham com sequentes ${ }^{1}$ ), e, por outro, os sistemas $\mathrm{Q}_{1} \mathrm{e}$ $\mathrm{Q}_{2}$, de Andréa Loparić ${ }^{2}$, que enfrentam o desafio de interpretar, sob um ponto de vista estritamente formal, as fórmulas de sexuação, de Lacan. Tive como intuito melhor compreender o funcionamento interno desses sistemas. Para tanto, abracei o projeto de normalizar o sistema dedutivo $S$, construído para $\mathrm{Q}_{1 \mathrm{p}}$ (extensão conservativa de $\mathrm{Q}_{1}$ ) por Fernando Paulo Christe Adorno ${ }^{3}$, apresentando um sistema de Gentzen coextensional a S. Esse é o resultado principal deste trabalho.

A opção por trabalhar com um sistema de dedução lógica ${ }^{4}$, de Gentzen, na normalização de $S$, ao invés de trabalhar com um sistema de dedução natural, merece algumas palavras. Isso porque, sendo $S$ um sistema de dedução natural, uma sua normalização em outro sistema de dedução natural permitiria preservar as propriedades dedutivas de S. Contudo, a possibilidade de que a tentativa de normalizar um sistema de dedução natural em um sistema de dedução lógica encerrasse maiores problemas e dificuldades, sobretudo no concernente à preservação das propriedades dedutivas do sistema de dedução natural, atraiu-me bastante.

\footnotetext{
${ }^{1}$ Cf. Gentzen, Gerhard. Recherches sur La Déduction Logique. Trad. Robert Feys e Jean Ladrière. Paris: Presses Universitaires de France, 1955, pp. 41-107.

${ }^{2}$ Cf. LoPARIĆ, Andréa. Les Négations et Les Univers du Discours. In: Lacan avec Les Philosophes. Paris: Albin Michel, 1991, pp. 239-264.

${ }^{3}$ Cf. Adorno, Fernando Paulo Christe. Dois Sistemas de Lógica Não Reflexiva. Dissertação de Mestrado. São Paulo: Universidade de São Paulo, 2001.

${ }^{4}$ As expressões "dedução natural" e "dedução lógica" são utilizadas por Gentzen para distinguir, respectivamente, entre os sistemas NJ e NK, que admitem hipóteses em suas demonstrações, e os sistemas LJ e LK, cujas demonstrações partem, sempre, do sequente fundamental. Cf. GENTZEN, Ibid., pp. $4-5$ e 43.
} 
Gentzen demonstrou que seu sistema LK é equivalente ao cálculo clássico, nos moldes do formalismo de Hilbert ${ }^{5}$. Tal sistema, porém, não inclui o símbolo lógico da igualdade em seu vocabulário, razão pela qual foi preciso estender a linguagem de LK de modo a contemplar esse símbolo, bem como acrescentar um postulado ao sequente fundamental, e uma nova figura de dedução, para permitir que uma fórmula em que tal símbolo ocorra seja introduzida numa demonstração, e articulada com as demais fórmulas presentes nessa demonstração. Uma vez acrescidas a constante lógica da igualdade ao vocabulário de LK e a definição de fórmula em que tal constante ocorre à sintaxe desse sistema, o postulado acrescentado ao sequente fundamental foi o da introdução da identidade, e a figura de dedução acrescentada às demais figuras, a da substituição de idênticos. Além disso, foi preciso acrescentar um outro postulado aos já mencionados, o da introdução de objetos clássicos, e modificar a figura da introdução do universal no antecedente, de modo a tornar o sistema correto com respeito à semântica de $\mathrm{Q}_{1 \mathrm{p}}$ - logo, com respeito à semântica de $\mathrm{Q}_{1}$. As demais figuras de dedução se mantiveram tais quais no sistema LK, bem como o sequente fundamental.

O sistema resultante de tais modificações é o sistema $\mathrm{QG}_{1}$, apresentado na segunda parte desta dissertação. Na terceira parte, foi provada a equivalência entre esse sistema e o cálculo $\mathrm{S}$, desenvolvido por Adorno, cuja correção e completude relativamente ao sistema $\mathrm{Q}_{1 \mathrm{p}}$ já fora demonstrada ${ }^{6}$. Consequentemente, o sistema $\mathrm{QG}_{1}$ é, também, correto e completo relativamente ao sistema $\mathrm{Q}_{1}$. Logo, visto que $\mathrm{Q}_{1 \mathrm{p}}$ é uma extensão conservativa de $\mathrm{Q}_{1}$, no universo das fórmulas de $\mathrm{Q}_{1}$ valerão a completude e a correção de $\mathrm{QG}_{1}$. Ademais, $\mathrm{QG}_{1}$ é correto e completo relativamente ao sistema $\mathrm{Q}_{2}$, uma

\footnotetext{
${ }^{5}$ Cf. GentZen, Ibid., pp. 129-164.

${ }^{6} \mathrm{Cf}$. AdORNo, Ibid., pp. 72-78.
} 
vez que fora demonstrado por Adorno ${ }^{7}$ que o sistema $\mathrm{Q}_{2}$ é tradutível em $\mathrm{Q}_{1}-\mathrm{e}$, portanto, em $\mathrm{QG}_{1}$.

A primeira parte deste trabalho é um estudo detalhado dos sistemas $\mathrm{Q}_{1}$ e $\mathrm{Q}_{2}$, cujas semânticas admitem a possibilidade de algo que, malgrado não se mostre à linguagem como propriamente um objeto, esteja contudo no universo do discurso. Analiso, sobretudo, a natureza verbal desse "algo", pensado como uma ficção, bem como a reestruturação, nesses sistemas, do significado formal das fórmulas gerais em sua articulação com as negações - ou seja, das relações entre asserções sobre a existência ou inexistência de algo mediante sentenças existenciais e universais, com e sem negações.

\footnotetext{
${ }^{7}$ Cf.AdORNo, Ibid., pp. 79-80.
} 


\section{Os Sistemas $Q_{1}$ e $Q_{2}$}

\section{As fórmulas de sexuação}

Apresentados por Andréa Loparić em $1991^{8}$, os sistemas $\mathrm{Q}_{1}$ e $\mathrm{Q}_{2}$ têm por objetivo propor uma interpretação congruente, sob uma perspectiva lógica, às célebres (e controversas) fórmulas de sexuação, de Lacan. São quatro fórmulas, agrupadas em pares (um para o feminino, outro para o masculino), as quais se caracterizam por duas transgressões bastante significativas no uso dos símbolos da Lógica de Predicados Clássica de Primeira Ordem: as fórmulas do par feminino seriam, do ponto de vista sintático, expressões mal formadas da linguagem; além disso, uma vez corrigidas suas imperfeições materiais de modo a torná-las bem formadas, teriam, assim como as do par masculino, significados formais estranhos, visto que as fórmulas de cada um dos pares seriam contraditórias entre si, além de cada uma das fórmulas de cada um dos pares ser equivalente a exatamente uma das fórmulas do outro par.

Fórmulas Originais

\begin{tabular}{|c|c|}
\hline Masculino & Feminino \\
\hline$\exists x \overline{\Phi x}$ & $\bar{\exists} x \overline{\Phi x}$ \\
\hline$\forall x \Phi x$ & $\bar{\forall} x \Phi x$ \\
\hline
\end{tabular}

${ }^{8}$ LOPARIĆ, Andréa. Les Négations et Les Univers du Discours. In: Lacan avec Les Philosophes. Paris: Albin Michel, 1991, pp. 239-264. 
Fórmulas Corrigidas

\begin{tabular}{|c|c|}
\hline Masculino & Feminino \\
\hline$\exists x \overline{\Phi x}$ & $\overline{\exists x \overline{\Phi x}}$ \\
\hline$\forall x \Phi x$ & $\overline{\forall x \Phi x}$ \\
\hline
\end{tabular}

Obviamente, poder-se-ia objetar que Lacan se apropriara unicamente dos símbolos, dotando-lhes de um significado que muito pouco se aproximasse da interpretação propriamente lógica desses símbolos. As noções de existência, totalidade, negação, predicação e variável circulam entre áreas diversas, ganhando significados diversos quando recepcionadas em novos contextos. A utilização dos símbolos da Lógica seria, nesse caso, não mais do que um uso material, uma referência gráfica aos significados não formais encerrados nesses símbolos, sem uma relação rigorosa com as regras de construção de fórmulas da Lógica de Predicados Clássica de Primeira Ordem ou com os significados estritamente formais das fórmulas construídas mediante tais regras. Dizer, por exemplo, que "o fascismo é a negação da democracia" não significa determinar dois conjuntos complementares relativamente a um domínio clássico previamente dado, mas nada impediria que tal afirmação fosse apresentada, materialmente, por meio dos símbolos da igualdade e da barra superior:

$$
\text { Fascismo }=\overline{\text { Democracla }}
$$

Uma outra objeção diria respeito à intuição propriamente visual dos símbolos. Uma placa de trânsito que indica aos motoristas que determinada rua tem fluxo da esquerda para a direita é uma seta voltada para a direita, sem que tal seta remeta, nem mesmo de muito longe, ao significado da implicação material, usualmente denotado por 
uma seta voltada à direita. Se o desenho de tal placa for inserido na decoração de uma casa noturna, deixará de indicar a mão correta de direção em determinada rua (seu significado original), passando a compor um ambiente cujos estímulos visuais têm por finalidade algo muito diverso da finalidade original da placa. Nesse novo contexto, tanto mais congruente o desenho da placa de trânsito será com sua nova finalidade quanto se houver nele uma imperfeição, uma transgressão com respeito ao uso preciso dos símbolos nas placas de trânsito. Sob essa perspectiva, as fórmulas de sexuação poderiam ser pensadas como deliberadamente mal formadas - remetendo, assim, a uma finalidade outra que a de expressar conceitos por meio de signos: não a denotação de algo, mas a sugestão, por meio da intuição visual de seus desenhos, de significados não verbais 9 .

Tais objeções não esgotam, certamente, as muitas objeções possíveis a uma interpretação propriamente formal das fórmulas de sexuação. Contudo, seja qual for a interpretação proposta, não poderá dispensar a linguagem por meio da qual essas fórmulas foram construídas: a linguagem da Lógica de Predicados de Primeira Ordem. Isso significa assumir o fato evidente de que, para veicular seus significados, ainda que informais ou não verbais (ou quaisquer outros que se pretenda), foram utilizados como signos símbolos da Lógica de Predicados de Primeira Ordem, ordenados segundo a sintaxe dessa Lógica (mesmo que de modo imperfeito), e, ademais, foram tais sequências de signos chamadas de "fórmulas". Assim, dentre as muitas interpretações possíveis dessas fórmulas, sob as mais diversas perspectivas, coube aos sistemas $\mathrm{Q}_{1} \mathrm{e}$ $\mathrm{Q}_{2}$ prover uma interpretação propriamente formal, baseada no contexto original dos símbolos utilizados. À pergunta "Que significado tais fórmulas poderiam ter?"

\footnotetext{
${ }^{9}$ Sabe-se da importância que Lacan atribuía à comunicação não verbal na compreensão de sua doutrina. Ver, a esse respeito, ROUDINESCO, Elisabeth. Jacques Lacan: Esquisse d'Une Vie, Histoire d'Un Système de Pensée. Paris: Librairie Arthème Fayard, 1993.
} 
acrescentou-se a hipótese de que as fórmulas de sexuação fossem interpretadas sob uma perspectiva estritamente formal, resultando na pergunta "Se fossem interpretadas sob uma perspectiva estritamente formal, que significado tais fórmulas poderiam ter?"

O acréscimo de uma tal hipótese desloca o problema. Para os nossos fins, importa menos aos sistemas $\mathrm{Q}_{1}$ e $\mathrm{Q}_{2} \mathrm{o}$ que Lacan pretendera expressar por meio de suas fórmulas do que as possibilidades expressivas da linguagem mediante a qual foram construídas, no plano de uma extensão propriamente formal dessa linguagem. Isso significa dizer que, mais do que formas de expressar os significados das fórmulas de sexuação no contexto da teoria psicanalítica de Lacan, os sistemas $\mathrm{Q}_{1}$ e $\mathrm{Q}_{2}$ reformularam certos significados lógicos de modo a permitir que tais sequências de símbolos constituíssem teses de teorias formais de Primeira Ordem. Se $\mathrm{Q}_{1}$ e $\mathrm{Q}_{2}$ se relacionam ou não com a doutrina de Lacan (e, caso se relacionem, em que medida), é uma outra questão, da qual não nos ocuparemos neste trabalho.

Assumir a hipótese de que tais fórmulas possam ser interpretadas num plano estritamente formal, no entanto, exige uma nova hermenêutica. Isso porque as fórmulas de sexuação pertencentes ao par feminino, tal como originalmente construídas, não seriam, sequer, fórmulas, caso fossem lidas segundo as convenções usuais da linguagem para o uso da barra superior na Lógica de Predicados de Primeira Ordem. O problema que se apresenta envolve o núcleo daquilo que foi tomado como objeto de estudo (as sequências de símbolos que constituem as fórmulas de sexuação), e seu enfrentamento significa responder uma pergunta que, no plano em que se desenrola a resposta, simplesmente não tem sentido. Consequentemente, não resta outra alternativa senão dotá-la de sentido antes de (e para que se possa) respondê-la. Esta a razão pela qual as sequências de símbolos originalmente apresentadas por Lacan para as fórmulas de sexuação do par feminino foram corrigidas, na construção dos sistemas $Q_{1}$ e $Q_{2}$, de 
modo a torná-las conformes à definição usual de fórmula na Lógica de Predicados de Primeira Ordem.

A correção aplicada ao par feminino das fórmulas originais de Lacan, a óbvia consequência de interpretar não mais, estritamente, as seqüências de símbolos que compunham essas fórmulas, tanto quanto a opção de interpretar, sob uma perspectiva estritamente formal, expressões que foram quase certamente construídas para ser interpretadas em outro campo de conhecimento, têm, contudo, um caráter menos arbitrário do que possa parecer.

Noam Chomsky ${ }^{10}$, entre outros, assinala que as construções verbais, quaisquer que sejam, são interpretadas, sempre, de modo a fazer sentido no contexto em que são recebidas, independentemente do sentido almejado no contexto em que foram enviadas. A comunicação objetiva ocorre, de fato, quando há intersecção bastante entre esses contextos, permitindo que o sentido se mantenha suficientemente intacto na transmissão das construções verbais em questão para garantir a compreensão, por parte de quem recebe a mensagem, do sentido veiculado por quem a emitiu. Nessa medida, uma mensagem mal formulada, sob a perspectiva de quem a recebe, é reconstruída imediatamente para que possa ser compreendida, ainda que, nessa reconstrução, e da perspectiva de quem emitiu a mensagem, a mensagem compreendida eventualmente não mais corresponda à mensagem enviada.

Chomsky, no entanto, refere-se a contextos subjetivos e sociais. Isso não impede que o procedimento de interpretação seja o mesmo no caso dos sistemas $\mathrm{Q}_{1}$ e $\mathrm{Q}_{2}$ relativamente às fórmulas de sexuação, cujos contextos são teóricos. Tanto a correção das fórmulas originais do par feminino quanto a interpretação das fórmulas de ambos os

\footnotetext{
${ }^{10}$ Cf. CHOMSKY, Noam. Language and Mind. New York: Harcourt, Brace \& World, 1968. Faço tais observações a título de comentário geral, visto que uma análise detalhada do tema fugiria completamente ao escopo deste trabalho.
} 
pares sob uma perspectiva estritamente formal reconstroem, nos planos da sintaxe e da semântica, respectivamente, sentenças que de outra forma não teriam sentido no contexto estrito da Lógica de Predicados de Primeira Ordem. As negações, por exemplo, aplicam-se apenas a fórmulas inteiras; por não ser uma fórmula, um quantificador, tomado isoladamente, não pode ser negado. Além disso, não faz qualquer sentido a construção de duas teorias formais, ambas inconsistentes, e tais que a cada uma das duas teses apresentadas em uma corresponda, na outra, uma tese equivalente.

Examinemos, pois, como foram feitas as modificações necessárias para a obtenção de um leitura com sentido das fórmulas em causa.

\section{As Semânticas de $Q_{1}$ e $Q_{2}$}

Originalmente, $\mathrm{Q}_{1}$ e $\mathrm{Q}_{2}$ foram apresentados como sistemas semânticos cuja linguagem (vocabulário e sintaxe) é a do Cálculo de Predicados Clássico de Primeira Ordem com Identidade ${ }^{11}$. As semânticas de $\mathrm{Q}_{1}$ e $\mathrm{Q}_{2}$ diferem, tanto da semântica clássica quanto entre si, no que diz respeito à noção de satisfação para fórmulas gerais, e são constituídas, ambas, por modificações da semântica clássica que tornaram possível alterar, por um lado, a natureza da relação denotada pelo predicado lógico da identidade, e, por outro, a natureza dos valores passíveis de ser atribuídos a uma variável qualquer da linguagem por uma valoração. Para tanto, foi preciso distinguir entre o domínio de uma estrutura e o universo do discurso de uma linguagem nessa estrutura, postulando a existência potencial de algo que, mesmo não pertencendo ao domínio de tal estrutura, pudesse estar no âmbito do discurso. Essa distinção pode ser vista como uma distinção

\footnotetext{
${ }^{11}$ Cf. LOPARIĆ, Ibid., p. 243.
} 
entre linguagem e fato, considerando a possibilidade de que a linguagem extrapole o domínio dos fatos (portanto, o domínio da estrutura e das relações n-árias entre objetos dessa estrutura) para alcançar, ainda que por um viés oblíquo, algo que poderia ser de natureza verbal, sem qualquer referência factual: uma ficção.

As semânticas de $Q_{1}$ e $Q_{2}$ são obtidas a partir da semântica clássica por meio de algumas modificações centrais. A primeira consiste em definir uma interpretação como um par ordenado cujo primeiro elemento é, precisamente, a ficção a que nos referimos acima, chamada questão da interpretação - objeto que pode ou não pertencer ao domínio da estrutura em que se baseia tal interpretação -, e o segundo elemento é uma interpretação clássica baseada no domínio dessa estrutura. A segunda consiste em definir a relação de identidade como a diagonal do domínio da estrutura (isto é, como relação binária, entre objetos do domínio da estrutura, determinada pelos pares ordenados formados por um mesmo objeto), relação essa acrescida às demais relações n-árias entre objetos desse domínio que, juntamente com o próprio domínio, constituem a estrutura definida. Quando a questão pertence ao domínio da estrutura, temos uma interpretação que em nada difere da interpretção clássica. Quando, todavia, a questão não pertence ao domínio, a identidade não se aplica a ela. Assim, definir a relação de identidade entre objetos do domínio como diagonal da estrutura significa romper com a semântica clássica de Primeira Ordem, na medida em que a identidade deixa de ser uma relação inerente a qualquer objeto pertencente ao universo do discurso - ou seja, a identidade deixa de ser algo próprio de tudo o que pode ser alcançado como valor de uma variável da linguagem. Consequentemente, a definição da relação de identidade como diagonal do domínio da estrutura, que, na semântica clássica, coincide com a condição metalingüística de satisfação para fórmulas do tipo ' $\mathrm{x}=\mathrm{y}$ ', passa a ter, aqui, um outro significado, e o fato de que ' $\mathrm{x}=\mathrm{x}$ ' não vale mais universalmente evidencia que 
nem todo valor possível de uma variável deverá ser um "bom objeto". De fato, o que se quer obter é uma situação em que o domínio da estrutura não esgota o universo do discurso, ficando aberta a possibilidade de haver algo que não deva ser tratado, no plano da linguagem, como idêntico a si mesmo.

Assim, nos dois sistemas, teremos dois tipos admitidos de interpretação: as ditas “interpretações normais”, cujo conceito coincide com a noção clássica de interpretação, onde domínio e universo de discurso são o mesmo conjunto; e as interpretações não normais, onde o universo de discurso não se limita ao domínio da estrutura, mas engloba também a questão.

A questão da interpretação não é, de fato, necessariamente um objeto. Não pode, no plano da linguagem, ser tratada como necessariamente idêntica a si mesma, nem como numericamente distinta de qualquer outro objeto, seja este outro objeto um elemento do domínio, seja o que, por abuso de linguagem, diríamos uma "outra" questão. Se for um objeto submetido à identidade, a questão não será um objeto "a mais" no domínio, acrescentado a (e, portanto, distinto de) todos os outros objetos que a esse domínio "já pertenceriam”. Mas a questão pode não ser um objeto submetido à identidade e, nesse caso, não pertencerá ao domínio. Consequentemente, nesse último caso, a questão não integra qualquer relação n-ária entre objetos do domínio da estrutura (em particular, não pertence à relação unária que constitui o próprio domínio), razão pela qual situa-se no plano estritamente verbal. Por esse motivo, ela é introduzida separadamente, como o primeiro elemento do par ordenado que constitui uma interpretação para $\mathrm{Q}_{1}$ ou $\mathrm{Q}_{2}$, par ordenado esse que tem como segundo elemento uma interpretação clássica dos objetos do domínio e das relações n-árias entre objetos desse domínio. 
Se for um objeto, a questão não se distinguirá de algum objeto pertencente ao domínio da estrutura; será, portanto, esse objeto. Nesse caso, relembramos, a interpretação correspondente será clássica, uma vez que o domínio da estrutura coincidirá com o universo do discurso nessa interpretação, e tudo o que puder ser dito, no plano da linguagem, referir-se-á aos objetos desse domínio. Consequentemente, as semânticas de $\mathrm{Q}_{1}$ e $\mathrm{Q}_{2}$ não postulam a existência de uma ficção - em outras palavras: não postulam, a priori, que o universo do discurso seja, necessariamente, mais amplo que o domínio de uma estrutura dada. Admitem perfeitamente interpretações segundo as quais todos os objetos alcançados pela linguagem estejam bem definidos em termos de relações n-árias entre elementos do domínio, o universo do discurso coincida com esse domínio e as fórmulas de sexuação, de Lacan, sejam falsas. Admitem, portanto, os sistemas $\mathrm{Q}_{1}$ e $\mathrm{Q}_{2}$, interpretações que não constituam modelos para as fórmulas de sexuação.

Se não for um objeto clássico, a questão será alcançada por uma variável da linguagem; pertencerá, portanto, à união entre o domínio da estrutura e o conjunto formado pela própria questão. Tal união, contudo, pode ser classicamente descrita no plano da metalinguagem, onde a questão é tratada de modo usual, como objeto ${ }^{12}$. Nessa medida, a união entre o domínio da estrutura e a questão da interpretação se distingue do domínio da estrutura - e, portanto, no plano da metalinguagem, é possível definir o universo do discurso como um conjunto determinado por seus elementos. Esse conjunto é chamado sobredomínio da interpretação, e também se distingue perfeitamente do conjunto determinado pelos objetos pertencentes ao domínio da estrutura ${ }^{13}$ - chamado

\footnotetext{
${ }^{12}$ Dizer que a questão "não é idêntica a si mesma", ou que "não se distingue numericamente de outros objetos" significa diferenciá-la plenamente do conceito clássico de objeto. Nessa medida, sob a perspectiva da metalinguagem, o conceito de questão da interpretação, tanto quanto o de objeto, são ambos objetos bem definidos.

${ }^{13}$ No plano do conceito, a questão é um elemento que pertence ao sobredomínio de uma interpretação e, no entanto, não pertence ao domínio dessa interpretação.
} 
de domínio da interpretação. Nesse caso, haverá no sobredomínio algo que não pertence a nenhuma relação unária de objetos do domínio, nem a qualquer elemento de qualquer relação n-ária (para $n>1$ ) entre objetos do domínio; em particular, algo que não pertence nem mesmo à relação de identidade, tal como definida na estrutura. Isso equivale a dizer que a questão, no plano da linguagem, somente pode ser alcançada pela negação de predicados. Contudo, a possibilidade de haver algo que não pertença ao domínio da interpretação altera a extensão dessa negação, que passa a referir-se a tudo o que não esteja na extensão do predicado respectivo - portanto, não mais apenas ao complemento dessa extensão relativamente ao domínio. Como, do ponto de vista interno a uma teoria que admita a questão fora do domínio, não há como estabelecer a "quantidade da questão" - pode-se mostrar que tanto faz admitir um, dois ou até mesmo infinitos objetos fora do domínio -, o universo de discurso se apresenta menos como um conjunto que como uma multiplicidade não muito bem delimitada, digamos, um campo; da mesma forma, o complemento da extensão de um predicado qualquer da linguagem relativamente ao sobredomínio ${ }^{14}$ se apresentará, para a teoria, também como um campo. Assim, a própria noção de complemento se torna insuficiente para descrever as extensões de negações de predicados - extensões essas que, no entanto, claramente, incluem os complementos dos respectivos predicados ${ }^{15}$.

Do ponto de vista da metalinguagem, um campo é um subconjunto do sobredomínio da interpretação. Do ponto de vista interno a uma teoria, um campo engloba tudo aquilo de que se pode falar. Aqui, novamente, a natureza das semânticas de $\mathrm{Q}_{1}$ e $\mathrm{Q}_{2}$ se manifesta: caso a questão pertença ao domínio da interpretação, campos aparecerão como conjuntos, determinados univocamente pelos objetos que pertencem a eles; caso a questão não pertença ao domínio da interpretação, os campos incluirão

\footnotetext{
${ }^{14}$ Tal como definidos, na metalinguagem, os conceitos de complemento e sobredomínio.

${ }^{15}$ Cf. LOPARIĆ, Ibid., pp. 254-256.
} 
conjuntos (mesmo que, eventualmente, o conjunto vazio), mas se apresentarão de modo mais amplo e menos delimitado que tais conjuntos, abarcando referências da linguagem que não encontram referentes no plano dos objetos. Tais referências não se podem manifestar senão pela negação das expressões que se aplicam a objetos - portanto, não se podem manifestar senão pela negação de predicados. Assim, no contexto de uma interpretação que associe uma variável da linguagem à questão, as negações de predicados abarcarão toda e qualquer referência que esteja fora das extensões dos respectivos predicados, e não somente as extensões dos complementos dos mesmos com respeito ao domínio.

Em particular, a extensão da negação da fórmula ' $x=x$ ', numa interpretação não normal, não mais é o conjunto vazio. Tal extensão contém, certamente, o conjunto vazio, mas, nesse contexto, abarca também aquilo que escapa à referência objetual da linguagem - isto é, abarca o sobredomínio da interpretação. Consequentemente, assertar que algo seja diferente de si mesmo significará estabelecer que algo não é um elemento do domínio, não pertence a uma relação da estrutura, e, portanto, não poderá entrar na composição de um predicado. Isto poderá ser formulado mediante duas sentenças:

1) Existe algo que não é idêntico a si mesmo; ou

2) Nem tudo é idêntico a si mesmo.

De um ponto de vista clássico, tais sentenças são equivalentes. Sua distinção marca, porém, a distinção entre os sistemas $Q_{1}$ e $Q_{2}$, tanto quanto sua capacidade expressiva, ao distinguir entre os significados das quantificações existencial e universal não somente em termos de existência ou inexistência, respectivamente, de objetos pertencentes a um determinado domínio, mas em termos do alcance precisamente daquilo que ultrapassa qualquer domínio - e que, no entanto, pode ser alcançado pela 
linguagem. Marca, ainda, a consistência de cada um dos pares de fórmulas de sexuação apresentados por Lacan, tanto quanto a não equivalência, em cada um dos sistemas, entre cada uma das fórmulas de um par e exatamente uma das fórmulas do outro.

Para dar conta dessa distinção, os sistemas $\mathrm{Q}_{1}$ e $\mathrm{Q}_{2}$ apresentam modificações, cada um de maneira diversa, nos conceitos clássicos de satisfação para as fórmulas gerais. Tais modificações baseiam-se, por sua vez, no acréscimo dos conceitos de sobrevaloração e sobrevaloração normal ao conceito clássico de valoração ${ }^{16}$ : uma sobrevaloração é uma função que associa, a cada variável da linguagem, um elemento do sobredomínio da interpretação; é dita normal em $x$, se associa $x$ a um elemento do domínio da interpretação. Além disso, uma sobrevaloração $s^{\prime}$ é $x$-variante de uma sobrevaloração $s$ se $s^{\prime}$ associa, a cada uma das variáveis que não $x$, os mesmos objetos do sobredomínio associados a tais variáveis por $s$. Com fulcro nessas alterações, é possível definir a satisfação das fórmulas gerais de modo a prover significados diversos às quantificações "existe algo" e "nem tudo", quando têm como escopo a mesma fórmula, mediante a distinção entre as quantificações existencial e universal. Tais significados diversos permitem, por fim, distinguir entre as sentenças 1 e 2 , acima.

Dadas uma estrutura $A$, uma interpretação ? $\mathcal{J}$ da linguagem sobre $A$, uma variável, ' $x$ ', os quantificadores universal, ' $\forall$ ', e existencial, ' $\exists$ ', um predicado unário, ' $\phi$ ', uma sobrevaloração $s$ da linguagem sobre ? $\mathcal{J}$, e mantidas as definições clássicas de fórmula, verdade e, para as fórmulas atômicas e moleculares, satisfação ${ }^{17}$, os sistemas $\mathrm{Q}_{1}$ e $\mathrm{Q}_{2}$ permitem distinguir entre os significados das quantificações existencial e universal mediante as seguintes definições:

\footnotetext{
${ }^{16}$ Cf. LOPARIĆ, Ibid., p. 244.

${ }^{17}$ Cf. LOPARIĆ, Ibid., pp. 243-245.
} 
$\mathrm{Q}_{1}[\forall]$ : Uma sobrevaloração $s$ satisfaz a fórmula $\forall x \phi x$ ' se toda sobrevaloração $s^{\prime} x$-variante de se normal em $x$ satisfaz a fórmula ' $\phi x$ ';

$\mathrm{Q}_{1}[\exists]:$ Uma sobrevaloração $s$ satisfaz a fórmula ' $\exists x \phi x$ ' se há sobrevaloração $s^{\prime} x$-variante de $s$ tal que $s^{\prime}$ satisfaz a fórmula ' $\phi x$ '.

$\mathrm{Q}_{2}[\forall]$ : Uma sobrevaloração $s$ satisfaz a fórmula ' $\forall x \phi x$ ' se toda sobrevaloração $s^{\prime} x$-variante de $s$ satisfaz a fórmula ' $\phi x$ ';

$\mathrm{Q}_{2}[\exists]$ : Uma sobrevaloração $s$ satisfaz a fórmula ' $\exists x \phi x$ ' se há sobrevaloração $s^{\prime} x$-variante de $s$ e normal em $x$ tal que $s^{\prime}$ satisfaz a fórmula ' $\phi x$ '.

Tais definições, assim como as correções aplicadas às fórmulas do par feminino, podem ser vistas como sugeridas pelas transgressões presentes nas fórmulas de sexuação originais. Há uma simetria em tais fórmulas, marcada tanto pelo uso das quantificações quanto pelo das negações - precisamente os elementos semânticos alterados nos sistemas $\mathrm{Q}_{1}$ e $\mathrm{Q}_{2}$. Cada par dessas fórmulas é constituído por uma expressão na qual ocorre um quantificador existencial e uma expressão na qual ocorre um quantificador universal. No par masculino, há uma expressão que contém uma barra superior (um símbolo de negação...), e outra que não contém nenhuma barra superior; as expressões do par feminino resultam do acréscimo, às expressões do par masculino, de uma barra superior sobre o símbolo de quantificação respectivo. Assim, o par masculino é constituído por uma expressão que contém uma barra superior, enquanto a outra não contém nenhuma barra superior, e o par feminino é constituído por uma expressão que contém uma barra superior, enquanto a outra contém duas barras superiores. Tais características sintáticas, interpretadas sob um ponto de vista clássico, sugerem que as fórmulas de sexuação do par feminino sejam, precisamente, a negação das fórmulas do par masculino - interpretação que, no plano semântico, mantém a simetria: cada uma 
das fórmulas de cada um dos pares diz exatamente o contrário da outra fórmula do mesmo par; o que é dito por meio da fórmula que contém um quantificador existencial, num par, é exatamente o que é dito por meio da fórmula que contém o quantificador universal, no outro; por fim, o que é dito por meio da fórmula que contém uma barra superior, num par, é exatamente o que é dito por meio da fórmula que contém uma barra superior, no outro, e o que é dito por meio da fórmula que não contém nenhuma barra superior, num par, é exatamente o que é dito por meio da fórmula que contém duas barras superiores, no outro. Se, contudo, na semântica clássica de primeira ordem, cada um dos pares é formado por fórmulas contraditórias e, em cada um dos pares, há uma fórmula equivalente a uma fórmula do outro par, novamente as características sintáticas dessas fórmulas, por meio das transgressões no par feminino, sugerem que as quantificações existencial e universal, em sua articulação com as negações, sejam lidas sob uma perspectiva não clássica, distinguindo entre as extensões dessas quantificações, bem como entre as extensões de suas respectivas negações. Sugerem, ainda, que a simetria entre as fórmulas seja rearticulada. É justamente isto o que as semânticas de $\mathrm{Q}_{1}$ e $\mathrm{Q}_{2}$ perfazem.

Nos contextos de $\mathrm{Q}_{1}$ e $\mathrm{Q}_{2}$, as fórmulas de sexuação não mais serão contraditórias em cada um dos pares, nem cada uma dessas fórmulas será equivalente, em cada uma dessas semânticas, a uma fórmula do outro par. A fórmula existencial, no par masculino, que contém uma ocorrência do símbolo de negação, dirá exatamente o mesmo, em $\mathrm{Q}_{1}$, que a negação da fórmula universal, no par feminino, em $\mathrm{Q}_{2}$, que também contém uma ocorrência do símbolo de negação; a fórmula universal, no par masculino, que não contém ocorrência do símbolo de negação, dirá em $\mathrm{Q}_{1}$ exatamente o mesmo que a negação da fórmula existencial do par feminino em $\mathrm{Q}_{2}$, que contém duas ocorrências do símbolo de negação (exatamente como num contexto clássico). Por outro 
lado, tanto a fórmula existencial quanto a fórmula universal do par masculino dirão exatamente o contrário do que dizem suas respectivas negações, no par feminino, caso sejam todas interpretadas no contexto exclusivamente de $\mathrm{Q}_{1}$, ou de $\mathrm{Q}_{2}$ (exatamente como num contexto clássico). A simetria entre as fórmulas de sexuação é, portanto, reposta, mediante a simetria entre as semânticas de $\mathrm{Q}_{1}$ e $\mathrm{Q}_{2}$, como rearticulação das relações de contradição e equivalência entre essas fórmulas num contexto clássico: a cada uma das fórmulas de cada um dos pares corresponde, em cada um dos sistemas, exatamente uma que lhe é contraditória, e a cada uma das fórmulas de cada um dos pares corresponde, num sistema, exatamente uma fórmula do outro par, no outro sistema, que lhe é equivalente.

Assim, nos contextos de $\mathrm{Q}_{1}$ e $\mathrm{Q}_{2}$, as fórmulas de sexuação constituirão teorias formais dos sistemas $\mathrm{Q}_{1}$ (o par masculino) e $\mathrm{Q}_{2}$ (o par feminino), cujos modelos serão interpretações não normais (ou seja, onde a questão não pertence ao domínio da estrutura) nas quais o predicado $\phi$ é associado ao domínio da estrutura. Em particular, podemos entender $\phi$ como uma escrita alternativa para a diagonal do domínio - ou seja, nossa identidade. Dessa forma, teremos:

- $\exists x \neg x=x$ será verdadeira segundo ?J em $\mathrm{Q}_{1}$, pois há uma sobrevaloração $s^{\prime} x$-variante de qualquer sobrevaloração $s$ da linguagem tal que $s^{\prime}$ associa $x$ à questão de ${ }^{?} \mathcal{J}-\mathrm{e}$, portanto, $s^{\prime}$ não satisfaz $x=x$. Logo, pela definição de satisfação para fórmulas moleculares, $s^{\prime}$ satisfaz $\neg x=x$. Logo, toda sobrevaloração da linguagem satisfaz $\exists x \neg x=x$. Consequentemente, pela interpretação de $\phi$, toda sobrevaloração da linguagem satisfaz $\exists x \neg \phi x$; 
- $\forall x x=x$ será verdadeira segundo ? $\mathcal{J}$ em $\mathrm{Q}_{1}$, pois toda sobrevaloração $s^{\prime} x$-variante de qualquer sobrevaloração $s$ da linguagem é tal que, se $s^{\prime}$ é normal em $x$, então $s^{\prime}$ não associa $x$ à questão de ${ }^{?} \mathcal{J}$. Portanto, $s^{\prime}$ satisfaz $x=x$. Logo, toda sobrevaloração da linguagem satisfaz $\forall x x=x$. Consequentemente, pela interpretação de $\phi$, toda sobrevaloração da linguagem satisfaz $\forall x \phi x$;

- $\neg \exists x \neg x=x$ será verdadeira segundo $\quad{ }^{?} \mathcal{J}$ em $\quad \mathrm{Q}_{2}$, pois toda sobrevaloração $s^{\prime} x$-variante de qualquer sobrevaloração $s$ da linguagem é tal que, se $s^{\prime}$ é normal em $x$, então $s^{\prime}$ não associa $x$ à questão de ?J. Portanto, $s^{\prime}$ satisfaz $x=x$. Logo, pela definição de satisfação para fórmulas moleculares, $s^{\prime}$ não satisfaz $\neg x=x-\mathrm{e}$, consequentemente, nenhuma sobrevaloração da linguagem satisfaz $\exists x \neg x=x$. Assim, pela definição de satisfação para fórmulas moleculares, toda sobrevaloração da linguagem satisfaz $\neg \exists x \neg x=x$, e segue-se pela interpretação de $\phi$ que toda sobrevaloração da linguagem satisfaz $\neg \exists x \neg \phi x$;

- $\neg \forall x x=x$ será verdadeira segundo ? $\mathcal{J}$ em $\mathrm{Q}_{2}$, pois há uma sobrevaloração $s^{\prime} x$-variante de qualquer sobrevaloração $s$ da linguagem tal que $s^{\prime}$ associa $x$ à questão de ${ }^{?} \mathcal{J}-\mathrm{e}$, portanto, $s^{\prime}$ não satisfaz $x=x$. Logo, nenhuma sobrevaloração da linguagem satisfaz $\forall x x=x$. Consequentemente, pela definição de satisfação para fórmulas moleculares, toda sobrevaloração da linguagem satisfaz $\neg \forall x x=x$, e segue-se pela interpretação de $\phi$ que toda sobrevaloração da linguagem satisfaz $\neg \forall x \phi x$. 


\section{O sistema $\mathbf{Q}_{1 \mathrm{p}}$}

Em sua dissertação de mestrado, Fernando Paulo C. Adorno ${ }^{18}$ apresentou duas extensões cumulativas da semântica original de $\mathrm{Q}_{1}$, denominadas sistemas $\mathrm{Q}_{1}$ e $\mathrm{Q}_{1 \mathrm{p}}$. $\mathrm{A}$ primeira dessas extensões incorporou nomes e símbolos funcionais à linguagem de $\mathrm{Q}_{1}$, fazendo corresponder a esses nomes e símbolos funcionais, na semântica de $\mathrm{Q}_{1}$, objetos que pertencem ao domínio da estrutura e operações n-árias definidas nesse domínio, respectivamente. A segunda dessas extensões incorporou parâmetros à linguagem da primeira extensão, fazendo corresponder a esses parâmetros elementos do sobredomínio da estrutura, e, aos símbolos funcionais, operações n-árias entre elementos desse sobredomínio. Com base nesta segunda extensão, Adorno apresentou o cálculo dedutivo S, sistema de dedução natural correto e completo relativamente a $\mathrm{Q}_{1}$. Provou, afinal, em $S$, que o sistema $Q_{2}$ é definível em $Q_{1}$ - isto é, que a semântica de $Q_{2}$ (em particular, no que diz respeito à satisfação das fórmulas gerais) é expressável em $\mathrm{Q}_{1}$.

Visto que a extensão $\mathrm{Q}_{1 \mathrm{p}}$ contém a extensão $\mathrm{Q}_{1}$, e que o sistema dedutivo $\mathrm{S}$ foi construído para $\mathrm{Q}_{1 \mathrm{p}}$, descreveremos o sistema $\mathrm{Q}_{1 \mathrm{p}}$, no que se segue ${ }^{19}$.

Foi definida uma linguagem $\mathcal{L}^{\prime}$ para $\mathrm{Q}_{1 \mathrm{p}}$, cujo vocabulário é o seguinte:

a) Um conjunto infinito e enumerável de variáveis, $\left\{x, x_{1}, x_{2}, \ldots\right\}$;

b) As seguintes constantes lógicas:

i) O conectivo unário da negação, ' $\neg$ ';

ii) Os conectivos binários da conjunção, ' $\wedge$ ', disjunção, ' $\vee$ ', implicação material, 'د’ e bi-implicação material, ' $\leftrightarrow$ ';

iii) Os símbolos de quantificação existencial, ' $\exists$ ', e universal, ' $\forall$ ';

\footnotetext{
${ }^{18}$ ADORnO, Fernando Paulo Christe. Dois Sistemas de Lógica Não Reflexiva. Dissertação de Mestrado. São Paulo: Universidade de São Paulo, 2001.

${ }^{19}$ Cf. ADORNO, Ibid., pp. 10-16.
} 
iv) O símbolo de igualdade, '=';

c) As seguintes constantes não-lógicas:

i) Um conjunto infinito e enumerável de nomes, $\left\{a, a_{1}, a_{2}, \ldots\right\}$;

ii) Um conjunto infinito e enumerável de parâmetros, $\left\{p, p_{1}, \ldots\right\}$;

iii) Um conjunto infinito e enumerável de símbolos funcionais, $\left\{f^{1}, f_{1}^{1}\right.$, $\left.f_{2}^{1}, \ldots, f^{2}, f_{1}^{2}, f_{2}^{2}, \ldots\right\}$

iv) Um conjunto infinito e enumerável de símbolos de predicados, $\left\{P^{1}\right.$, $\left.P_{1}^{1}, P_{2}^{1}, \ldots, P^{2}, P_{1}^{2}, P_{2}^{2}, \ldots\right\}$

d) Os sinais de pontuação '(' e ')'.

As variáveis, nomes e parâmetros foram chamados símbolos individuais de $\mathcal{L}^{\prime}$; os nomes e parâmetros foram chamados pronomes.

A partir desse vocabulário, um pró-termo de $\mathrm{Q}_{1 \mathrm{p}}$ foi definida como a seguir:

a) Um símbolo individual de $\mathcal{L}^{\prime}$;

b) $\varphi_{i}^{n} \tau_{1} \ldots \tau_{n}$, onde $\varphi_{i}^{n}$ é um símbolo funcional e $\tau_{1}, \ldots, \tau_{n}$ são pró-termos quaisquer da linguagem.

As pró-fórmulas atômicas de $\mathrm{Q}_{1 \mathrm{p}}$ foram definidas como expressões da forma:

a) $\Pi_{i}^{n} \tau_{1}, \ldots, \tau_{n}$, onde $\Pi_{i}^{n}$ é um símbolo de predicado e $\tau_{1}, \ldots, \tau_{n}$ são pró-termos quaisquer da linguagem;

b) $\tau_{1}=\tau_{2}$, onde $\tau_{1}$ e $\tau_{2}$ são pró-termos quaisquer da linguagem.

A partir dessa base, foi definida pró-formula de $\mathrm{Q}_{1 \mathrm{p}}$ como a seguir:

a) Toda pró-fórmula atômica é uma pró-fórmula;

b) Se $\alpha$ e $\beta$ são pró-fórmulas quaisquer, então são pró-fórmulas moleculares as expressões da forma ' $\neg \alpha$ ', ' $(\alpha \wedge \beta)$ ', '( $\alpha \vee \beta)$ ', ' $(\alpha \supset \beta)$ ' e ' $(\alpha \leftrightarrow \beta)$ '; 
c) Se $Q$ é um símbolo de quantificação, $\S$ é uma variável e $\alpha$ é uma pró-fórmula qualquer, então $Q \S \alpha$ é uma fórmula geral (nestas, a sequência de símbolos $Q \S$ foi chamada um quantificador em $\alpha$, e $\alpha$ foi chamado o escopo do quantificador $Q \S)$.

Uma ocorrência ligada de uma variável $\S$ numa fórmula $\alpha$ foi definida como uma ocorrência de $\S$ que compõe um quantificador $Q \S$ em $\alpha$, ou que se encontra no escopo de um quantificador $Q \S$ em $\alpha$; caso contrário, tal ocorrência de $\S$ em $\alpha$ foi definida como livre.

Uma pró-sentença foi definida como uma pró-fórmula que não contêm ocorrência livre de variável.

Foi chamada ordem alfabética àquela induzida pela ordem crescente dos índices desses nomes.

Uma estrutura $A$ para $\mathrm{Q}_{1 \mathrm{p}}$ foi assim definida como:

a) Um conjunto não vazio, $|A|$ chamado domínio da estrutura;

b) A relação de identidade em $|A|,={ }_{A}$;

c) Um certo número de relações n-árias em $|A|$, para $n \geq 1$;

d) Um certo número de operações n-árias em $|A|$, para $n \geq 1$;

Uma pró-interpretação ${ }^{?} \mathcal{J}_{p}$ de $\mathcal{L}^{\prime}$ numa dada estrutura $A$ foi definida como o par ordenado $\left({ }^{?} \mathcal{J}, \mathcal{J}_{p}\right)$, onde:

a) ? J é um par ordenado $(? \mathcal{\jmath}, \mathcal{J})$, onde:

i) $?_{\mathfrak{J}}$, chamada a questão de ${ }^{?} \mathcal{J}_{p}$, é um elemento que pode ou não pertencer a $|A|$;

ii) $\quad \mathcal{J}$ é uma função que associa: 
1) A cada nome $c$, um objeto, $\mathcal{J}(c)$, tal que $\mathcal{J}(c) \in|A|$;

2) A cada símbolo funcional $\varphi_{i}^{n}$, uma operação n-ária, $\mathcal{J}\left(\varphi_{i}^{n}\right)$, em $|A|$

3) A cada símbolo de predicado $\Pi_{i}^{n}$, uma relação n-ária, $\mathcal{J}\left(\Pi_{i}^{n}\right)$, em $|A|$.

b) $\mathcal{J}_{p}$ é uma função que associa:

i) A cada nome $c$, o objeto $\mathcal{J}(c)$;

ii) A cada símbolo funcional $\varphi_{i}^{n}$, a operação n-ária $\mathcal{J}\left(\varphi_{i}^{n}\right)$;

iii) A cada símbolo de predicado $\Pi_{i}^{n}$, a relação n-ária $\mathcal{J}\left(\Pi_{i}^{n}\right)$;

iv) A cada parâmetro $\xi$, um objeto, $J_{p}(\xi)$, tal que $J_{p}(\xi) \in\left\{?_{\mathcal{J}}\right\} \cup|A|$.

O conjunto $\left\{?_{\jmath}\right\} \cup|A|$ foi chamado o sobredomínio de ${ }^{?} \mathcal{J}_{p}$.

Uma pró-interpretação ${ }^{?} \mathcal{J}_{p}$ de $\mathcal{L}^{\prime}$ numa dada estrutura $A$ foi dita pró-completa se, para cada um dos elementos do sobredomínio de ${ }^{?} \mathcal{J}_{p}$, existe um pronome $\mathcal{L}^{\prime}$ associado a esse elemento por ${ }^{?} \mathcal{J}_{p}$.

Uma sobrevaloração $s$ numa estrutura $A$ com respeito a uma pró-interpretação ${ }^{?} \mathcal{J}_{p}$ de $\mathcal{L}^{\prime}$ foi definida como uma função que associa as variáveis de $\mathcal{L}^{\prime}$ a elementos do sobredomínio de ${ }^{?} \mathcal{J}_{p}$. Tal sobrevaloração foi dita normal em $\S$ se $s(\S) \in|A|$.

Duas sobrevalorações quaisquer $s$ e $s^{\prime}$ foram ditas $\S$-variantes se, para todo $\S^{\prime}$ tal que $\S^{\prime} \neq \S, s\left(\S^{\prime}\right)=s^{\prime}\left(\S^{\prime}\right)$

Dadas uma pró-interpretação ${ }^{?} \mathcal{J}_{p}$ de $\mathcal{L}^{\prime}$ numa estrutura $A$ e uma sobrevaloração $s$ em $A$ com respeito a ${ }^{?} \mathcal{J}_{p}$, a função preenchimento por ${ }^{?} \mathcal{J}_{p}$ foi definida como a função 
que associa, a cada pró-termo $\tau$ de $\mathcal{L}^{\prime}$, um elemento $S_{\mathcal{J}_{p}}(\tau)$ do sobredomínio de ${ }^{?} \mathcal{J}_{p}$ tal que:

a) Se $\tau$ é uma variável, então $s_{\jmath_{p}}(\tau)$ é $s(\tau)$;

b) Se $\tau$ é um pronome, então $S_{\jmath_{p}}(\tau)$ é $\mathcal{J}_{p}(\tau)$;

c) Se $\tau$ é da forma $\varphi_{i}^{n} \tau_{1} \ldots \tau_{n}$, então:

i) $\quad s_{\jmath_{p}}(\tau)$ é $\mathcal{J}_{p}\left(\varphi_{i}^{n}\right) s_{\jmath_{p}}\left(\tau_{1}\right) \ldots s_{\jmath_{p}}\left(\tau_{n}\right)$, se para todo $k(1 \leq k \leq n)$, $s_{\jmath_{p}}\left(\tau_{k}\right) \in|A|$

ii) $\quad S_{\jmath_{p}}(\tau)$ é $?_{\mathcal{J}}$, caso contrário.

Dadas uma pró-interpretação ${ }^{?} \mathcal{J}_{p}$ de $\mathcal{L}^{\prime}$ numa estrutura $A$, uma sobrevaloração $s$ em $A$ com respeito a ${ }^{?} \mathcal{J}_{p}$ e uma pró-fórmula $\alpha$, foi definido que $s$ satisfaz $\alpha$ segundo ${ }^{?} \mathcal{J}_{p}$ se:

a) $\alpha$ é atômica, e:

i) $\quad \alpha$ é $\Pi_{i}^{n} \tau_{1}, \ldots, \tau_{n}, \mathrm{e}\left(s_{\jmath_{p}}\left(\tau_{1}\right) \ldots s_{\jmath_{p}}\left(\tau_{n}\right)\right) \in \mathcal{J}_{p}\left(\Pi_{i}^{n}\right)$;

ii) $\quad \alpha$ é $\tau_{1}=\tau_{2}$, e $\left(s_{\jmath_{p}}\left(\tau_{1}\right), s_{\jmath_{p}}\left(\tau_{2}\right)\right) \in={ }_{A}$;

b) $\alpha$ é molecular, e:

i) $\quad \alpha$ é $\neg \beta$, e $s$ não satisfaz $\beta$ segundo ${ }^{?} \mathcal{J}_{p}$;

ii) $\quad \alpha$ é $\left(\beta_{1} \wedge \beta_{2}\right)$, e $s$ satisfaz tanto $\beta_{1}$ quanto $\beta_{2}$ segundo ${ }^{?} \mathcal{J}_{p}$;

iii) $\quad \alpha$ é $\left(\beta_{1} \vee \beta_{2}\right)$, e $s$ satisfaz $\beta_{1}$ ou $\beta_{2}$ segundo ${ }^{?} \mathcal{J}_{p}$;

iv) $\quad \alpha$ é $\left(\beta_{1} \supset \beta_{2}\right)$, e $s$ não satisfaz $\beta_{1}$ ou satisfaz $\beta_{2}$ segundo ${ }^{?} \mathcal{J}_{p}$;

v) $\quad \alpha$ é $\left(\beta_{1} \leftrightarrow \beta_{2}\right)$, e $s$ satisfaz tanto $\beta_{1}$ quanto $\beta_{2}$ segundo ? $\mathcal{J}_{p}$ ou não satifaz nem $\beta_{1}$ nem $\beta_{2}$ segundo ${ }^{?} \mathcal{J}_{p}$; 
c) $\alpha$ é geral, e:

i) $\quad \alpha$ é $\forall \S \beta$, e qualquer sobrevaloração $s^{\prime} \S$-variante de $s$ normal em $\S$ satisfaz $\beta$ segundo ${ }^{?} \mathcal{J}_{p}$;

ii) $\quad \alpha$ é $\exists \S \beta$, e há sobrevaloração $s^{\prime} \S$-variante de $s$ tal que $s^{\prime}$ satisfaz $\beta$ segundo ${ }^{?} \mathcal{J}_{p}$.

Uma pró-sentença qualquer $\alpha$ foi definida como verdadeira segundo ${ }^{?} \mathcal{J}_{p}$ se qualquer sobrevaloração em $A$ com respeito a ${ }^{?} \mathcal{J}_{p}$ satisfaz $\alpha$, e como falsa segundo ${ }^{?} \mathcal{J}_{p}$ se nenhuma sobrevaloração em $A$ com respeito a ${ }^{?} \mathcal{J}_{p}$ satisfaz $\alpha$; além disso, $\alpha$ foi definida como pró-válida logicamente se é verdadeira segundo qualquer próinterpretação, e como pró-consequência lógica de um conjunto qualquer de prósentenças, $\Gamma$, se, em qualquer pró-interpretação ${ }^{?} \mathcal{J}_{p}$, toda sobrevaloração que satisfaz todas as pró-sentenças de $\Gamma$ segundo ${ }^{?} \mathcal{J}_{p}$ também satisfaz $\alpha$ segundo ${ }^{?} \mathcal{J}_{p}$.

A definição de consequência tautológica de uma pró-sentença a partir de um conjunto de pró-sentenças foi mantida clássica em $\mathrm{Q}_{1 \mathrm{p}}$.

Para nossos propósitos, cabe especial atenção às noções de parâmetro e função preenchimento por ${ }^{?} \mathcal{J}_{p}$.

Os parâmetros são artifícios da linguagem que permitem tratar a questão de ${ }^{?} \mathcal{J}_{p}$ como se fosse um objeto ${ }^{20}$. Desempenham, no plano da linguagem de $\mathrm{Q}_{1 \mathrm{p}}$, um papel semelhante ao desempenhado por expressões como "algo", ou "alguma coisa", na linguagem natural. É possível, com efeito, dizer que "algo não é idêntico a si mesmo", ou que "duas coisas não idênticas a si mesmas não podem ser nomeadas". Tais expressões não são propriamente nomes; visam, justamente, referir-se a objetos do

\footnotetext{
${ }^{20}$ Cf. ADORNO, Ibid., p. 7.
} 
universo do discurso sem que seja preciso nomeá-los - ou referir-se a esses objetos quando nomeá-los é impossível. De um ponto de vista clássico, "algo" e "alguma coisa" (tanto quanto a questão de ${ }^{?} \mathcal{J}_{p}$ ) se referem a objetos, funcionando como espécies de variáveis que, ao invés de admitir quaisquer objetos do domínio, admitem objetos dotados de certas propriedades ${ }^{21}$. No contexto de $\mathrm{Q}_{1 \mathrm{p}}$, os parâmetros estão associados a elementos do sobredomínio de ${ }^{?} \mathcal{J}_{p}$ - portanto, a elementos que podem ou não ser objetos, por um lado, e, por outro, que podem ou não ser nomeáveis no plano da linguagem.

A função preenchimento por ${ }^{?} \mathcal{J}_{p}$ garante que, no plano da metalinguagem, dada uma operação n-ária sobre elementos do sobredomínio de uma estrutura, se a questão da interpretação for um dos argumentos dessa operação, então será também o valor dessa operação. Isso significa não ser possível admitir operações que tratem, no plano dos fatos, objetos e ficções da mesma maneira: os elementos do sobredomínio da estrutura dada são objetos somente no plano verbal, e somente neste plano podem ser argumentos e valores de funções; no plano dos fatos, escapam à univocidade da função, visto que poderiam ser muitos e um só ao mesmo tempo, tanto como argumentos quanto como valores de uma determinada operação. Consequentemente, não podem estar associados (ou constituir, com outros objetos ou ficções, n-uplas ordenadas que estejam associadas) a objetos por nenhuma operação, nem objetos podem a eles estar associados (ou constituir, com outros objetos, n-uplas ordenadas que estejam a eles associadas). Em particular, isso significa que as ficções (ou seja, que a questão, ou questões de ${ }^{?} \mathcal{J}_{p}$ ) não podem ser indexadas, visto que não podem ser valores de uma função que tenha como

\footnotetext{
${ }^{21}$ Smullyan utiliza este artifício na terceira parte de First-Order Logic, para quantificar prefixos de configurações sem fazer incidir tais configurações sobre objetos quaisquer, mas sobre objetos com determinadas propriedades. Cf. Smullyan, Raymond M. First-Order Logic. New York: Dover, 1968, pp. 146-147.
} 
domínio o conjunto dos números naturais, do que decorre que não se distinguem numericamente.

Em razão da função preenchimento por ${ }^{?} \mathcal{J}_{p}$, um pró-termo qualquer da linguagem que tenha como argumento a questão da pró-interpretação estará associado, recursivamente, à própria questão da pró-interpretação. Logo, se uma pró-interpretação for pró-completa, haverá um parâmetro associado por essa pró-interpretação à denotação de qualquer pró-termo que tenha como argumento a questão da próinterpretação, tanto quanto haverá pronomes associados por essa pró-interpretação à denotação de qualquer pró-termo que tenha como argumentos apenas objetos do domínio. Isso permitirá que dispensemos os símbolos funcionais em nossa normalização do cálculo dedutivo $\mathrm{S}$, tratando os termos como variáveis, nomes ou parâmetros da linguagem sem, com isso, prejudicar a coextensão de nosso sistema com o sistema $\mathrm{S}$.

\section{O sistema $S$}

Apresentado como um sistema de dedução natural para $\mathrm{Q}_{1 \mathrm{p}}$, o sistema $\mathrm{S}$ é constituído pela definição de pró-dedução, base a partir da qual é possível estender, recursivamente, uma pró-dedução dada com $n$ linhas $(n \geq 1)$ a uma pró-dedução com $n+1$ linhas mediante 11 regras de inferência. Dessas regras, as mais significativas, no que diz respeito à semântica de $\mathrm{Q}_{1 \mathrm{p}}$, são as da Introdução de Axiomas $2\left(\mathbf{A x}_{\mathbf{2}}\right)$ e da Instanciação Universal (IU). A primeira, porque garante que a questão não seja alcançada por pró-sentenças atômicas, e, portanto, que nada se poderá predicar positivamente da questão. A segunda, porque garante que a questão não seja instância de nenhuma sentença universal, refletindo, assim, a noção de satisfação apresentada para as sentenças universais em $\mathrm{Q}_{1 \mathrm{p}}$. 
Uma pró-dedução, em $\mathrm{S}$, foi definida como uma sequência finita de linhas $l_{1}, l_{2}, \ldots, l_{n}$, cada uma das quais tem a forma $\mu(i) \alpha$, onde $i$ é o número da linha (isto é, o número natural que identifica a linha), $\alpha$ é a afirmação da linha $i$ (portanto, uma prósentença) e $\mu$ é o conjunto das dívidas da linha $i$ (isto é, um conjunto de números de linhas não superiores a $i$ ).

Uma pró-sentença $\beta$ foi dita mencionada em $\mu$ se $\beta$ é a afirmação de uma linha $i$ numa pró-dedução e $i$ pertence ao conjunto $\mu$ das dívidas de uma linha $j$ dessa mesma pró-dedução.

As seguintes regras permitem estender uma pró-dedução $D$ em $\mathrm{S}$ com $j-1$ linhas $(j \geq 1)$ a uma pró-dedução $D^{\prime}$ em $\mathrm{S}$ com $j$ linhas, acrescentando-se:

a) Por meio da regra $\mathbf{P}$, a linha $\{j\}(j) \alpha$, onde $\alpha$ é uma pró-sentença qualquer;

b) Por meio da regra $\mathbf{A} \mathbf{x}_{1}$, a linha $\emptyset(j) a=a$;

c) Por meio da regra $\mathbf{A} \mathbf{x}_{2}$, a linha $\emptyset(j) \alpha \supset \xi=\xi$, se $\alpha$ é atômica e $\xi$ ocorre em $\alpha$

d) Por meio da regra $\mathbf{T}$, a linha $\mu_{1} \cup \ldots \cup \mu_{n}(j) \beta$, se $\beta$ é consequência tautológica de $\left\{\alpha_{1}, \ldots \alpha_{n}\right\}$ e se ocorrem, em $D$, as linhas $\mu_{1}\left(i_{1}\right) \alpha_{1}, \ldots, \mu_{n}$ $\left(i_{n}\right) \alpha_{n}$

e) Por meio da regra $\mathbf{C}$, a linha $\mu(j) \alpha \supset \beta$, se ocorrem, em $D$, as linhas $\left\{i_{1}\right\}$ $\left(i_{1}\right) \alpha$ e $\mu \cup\left\{i_{1}\right\}\left(i_{2}\right) \beta$

f) Por meio da regra IU, a linha:

i) $\quad \mu(j) \alpha(\tau)$, se ocorre, em $D$, a linha $\mu(i) \forall \S \alpha$, se $\tau$ é um pró-termo em que não ocorrem variáveis nem parâmetros, e onde $\alpha(\tau)$ é o resultado da substituição de todas as ocorrências de $\S$ em $\alpha$ por ocorrências de $\tau$; 
ii) $\quad \mu \cup \mu_{1} \cup \ldots \cup \mu_{n}(j) \alpha(\tau)$, se ocorrem, em $D$, as linhas $\mu(i) \forall \S \alpha, \mu_{1}$ $\left(i_{1}\right) \xi_{1}=\xi_{1}, \ldots, \mu_{n}\left(i_{n}\right) \xi_{n}=\xi_{n}$, se $\tau$ é um pró-termo em que não ocorrem variáveis, se $\xi_{1}, \ldots, \xi_{n}$ são os parâmetros que ocorrem em $\tau$, e onde $\alpha(\tau)$ é o resultado da substituição de todas as ocorrências de $\S$ em $\alpha$ por ocorrências de $\tau$;

g) Por meio da regra $\mathbf{G U}$, a linha $\mu(j) \forall \S \alpha$, se ocorre, em $D$, a linha $\mu(i) \alpha(\sigma)$, se $\sigma$ é um pronome que não ocorre em $\alpha$ nem nas pró-sentenças mencionadas em $\mu$, e onde $\alpha(\tau)$ é o resultado da substituição de todas as ocorrências de $\S$ em $\alpha$ por ocorrências de $\tau$;

h) Por meio da regra IE, a linha $\mu_{1} \cup \mu_{3}(j) \beta$, se ocorrem, em $D$, as linhas $\mu_{1}$ $\left(i_{1}\right) \exists \S \alpha,\left\{i_{2}\right\}\left(i_{2}\right) \alpha(\xi), \mu_{3} \cup\left\{i_{2}\right\}\left(i_{3}\right) \beta$, se $\xi$ é um parâmetro que não ocorre em $\alpha$, nem em $\beta$, nem nas pró-sentenças mencionadas em $\mu_{3}$, e onde $\alpha(\xi)$ é o resultado da substituição de todas as ocorrências de $\S$ em $\alpha$ por ocorrências de $\xi$

i) Por meio da regra GE, a linha $\mu(j) \exists \S \alpha$, se ocorre, em $D$, a linha $\mu(i) \alpha(\tau)$, se $\tau$ é um pró-termo em que não ocorrem variáveis, e onde $\alpha(\tau)$ é o resultado da substituição de todas as ocorrências de $\S$ em $\alpha$ por ocorrências de $\tau$;

j) Por meio da regra $\mathbf{E}$, a linha $\mu(j) \exists \S \alpha$, se ocorre, em $D$, a linha $\mu(i)$ $\neg \forall \S \neg \alpha ;$

k) Por meio da regra $\mathbf{I}$, a linha $\mu_{1} \cup \mu_{2}(j) \beta$, se ocorrem, em $D$, as linhas $\mu_{1}\left(i_{1}\right)$ $\tau_{1}=\tau_{2},\left\{\mu_{2}\right\}\left(i_{2}\right) \alpha$, se $\tau_{1}$ e $\tau_{2}$ são pró-termos em que não ocorrem variáveis e se $\beta$ é o resultado da substituição de uma ou mais ocorrências de $\tau_{1}$, em $\alpha$, por ocorrências de $\tau_{2}$. 


\section{O Sistema $\mathbf{Q G}_{1}$}

\section{Linguagem}

A linguagem de $\mathrm{QG}_{1}$ é formada por:

e) Um conjunto infinito e enumerável de variáveis, $\left\{x, x_{1}, x_{2}, \ldots\right\}$;

f) As seguintes constantes lógicas:

v) O conectivo unário da negação, ' $\neg$ ';

vi) Os conectivos binários da conjunção, ' $\Lambda$ ', disjunção, ' $\mathrm{V}$ ', e implicação material, 'د';

vii) Os símbolos de quantificação existencial, ' $\exists$ ', e universal, ' $\forall$ ';

viii) O símbolo de igualdade, '=’;

g) As seguintes constantes não-lógicas:

v) Um conjunto infinito e enumerável de nomes, $\left\{a, a_{1}, a_{2}, \ldots\right\}$;

vi) Um conjunto infinito e enumerável de parâmetros, $\left\{p, p_{1}, \ldots\right\}$;

vii) Um conjunto infinito e enumerável de símbolos de predicados, $\left\{P^{1}\right.$, $\left.P_{1}^{1}, P_{2}^{1}, \ldots, P^{2}, P_{1}^{2}, P_{2}^{2}, \ldots\right\}$

h) Os seguintes simbolos auxiliares:
i) Parênteses esquerdo, '(', e direito, ')';
ii) O símbolo de implicação sequencial, ' $\rightarrow$,22.

\footnotetext{
${ }^{22}$ Sigo, aqui, a notação original de Gentzen (cf. GENTZEN, Gerhard. Recherches sur La Déduction Logique. Trad. Robert Feys e Jean Ladrière. Paris: Presses Universitaires de France, 1955, p.8).
} 


\section{Sintaxe}

Um termo será um nome, parâmetro ou variável da linguagem. Um nome ou parâmetro será dito um pronome, ou termo fechado, e uma variável, um termo aberto.

São fórmulas atômicas as expressões da forma:

c) $\Pi_{i}^{n} \tau_{1}, \ldots, \tau_{n}$, onde $\Pi_{i}^{n}$ é um símbolo de predicado e $\tau_{1}, \ldots, \tau_{n}$ são termos quaisquer da linguagem;

d) $\tau_{1}=\tau_{2}$, onde $\tau_{1}$ e $\tau_{2}$ são termos quaisquer da linguagem.

A partir dessa base:

d) Toda fórmula atômica é uma fórmula;

e) Se $\alpha$ e $\beta$ são fórmulas, então são também fórmulas as expressões da forma ‘ $\neg \alpha$ ', ' $(\alpha \wedge \beta)$ ', '( $\alpha \vee \beta)$ ', e '( $\alpha \supset \beta)$ ' (tais expressões são ditas fórmulas moleculares);

f) Se $Q$ é um símbolo de quantificação, $§$ é uma variável e $\alpha$ é uma fórmula, então $Q \S \alpha$ é também uma fórmula (tais expressões são ditas fórmulas gerais; nestas, a sequência de símbolos $Q \S$ será dita quantificador em $\alpha$, e $\alpha$ será dita escopo do quantificador $Q \S)$.

Uma ocorrência ligada de uma variável § numa fórmula $\alpha$ é uma ocorrência de $\S$ que compõe um quantificador $Q \S$ em $\alpha$, ou que se encontra no escopo de um quantificador $Q \S$ em $\alpha$; caso contrário, trata-se de uma ocorrência livre de $\S$ em $\alpha$.

Uma sentença é uma fórmula que não contêm ocorrência livre de variável.

A ordem alfabética dos nomes é aquela induzida pela ordem crescente dos índices desses nomes. 
Se $\Gamma$ é a sequência de fórmulas $\left\{\beta_{1}, \ldots, \beta_{n}\right\}$ e $\alpha$ é uma fórmula (que pode ou não pertencer a $\Gamma$ ), então:

a) $\Gamma, \alpha$ é a sequência de fórmulas $\left\{\beta_{1}, \ldots, \beta_{n}, \beta_{n+1}\right\}$, onde $\beta_{n+1}=\alpha$;

b) $\alpha, \Gamma$ é a sequência de fórmulas $\left\{\alpha_{1}, \ldots, \alpha_{n}, \alpha_{n+1}\right\}$, onde $\alpha_{1}=\alpha$ e, para cada $i, 1 \leq i \leq n, \alpha_{i+1}=\beta_{i}$

Se $\Gamma$ é a sequência de fórmulas $\left\{\beta_{1}, \ldots, \beta_{n}\right\}$ e $\Delta$ é é a sequência de fórmulas $\left\{\beta_{1}^{\prime}, \ldots, \beta_{m}^{\prime}\right\}$, então $\Gamma, \Delta$ é a sequência de fórmulas $\left\{\beta_{1}, \ldots, \beta_{n}, \beta_{n+1}, \ldots, \beta_{n+m}\right\}$, onde $\beta_{n+1}=\beta_{1}^{\prime}$ e $\beta_{n+m}=\beta_{m}^{\prime}$

Se $\Gamma$ e $\Delta$ são sequências finitas (e possivelmente vazias) de fórmulas, então $\Gamma \rightarrow \Delta$ será dito um sequente; $\Gamma$ será dito o antecedente, e $\Delta$, o consequente, do sequente $\Gamma \rightarrow \Delta$.

Uma figura de dedução é uma articulação entre sequentes que tem a seguinte forma:

$$
\frac{\mathfrak{S}_{1} \ldots \mathfrak{S}_{n}}{\mathfrak{I}}, \text { para } n \geq 1
$$

onde os sequentes $\mathfrak{S}_{1}, \ldots, \mathfrak{S}_{n}$ são ditos sequentes superiores da figura, enquanto o sequente $\mathfrak{I}$ é dito sequente inferior da figura.

Uma derivação consiste em um certo número de sequentes (pelo menos um) que formam entre si figuras de dedução da seguinte maneira:

a) Todo sequente é o sequente inferior de, no máximo, uma figura de dedução;

b) Todo sequente (com exceção de um único, o sequente final) é o sequente superior de, no mínimo, uma figura de dedução; 
c) Se um sequente não é sequente inferior de nenhuma figura de dedução, então este sequente é um posulado (ou seja, toda série de sequentes, numa derivação, tem início com um postulado).

Se o sequente $\Gamma \rightarrow \alpha$ for o sequente final de uma derivação, diremos que há uma derivação do sequente $\Gamma \rightarrow \alpha$.

Diremos que uma sentença $\alpha$ é derivável a partir de um conjunto $\Gamma$ de sentenças se houver uma derivação do sequente $\Gamma \rightarrow \alpha$.

Para o que se segue, fixaremos a seguinte notação:

a) letras gregas minúsculas, com ou sem subscritos ou sobrescritos, signifcarão sentenças;

b) letras gregas maiúsculas, com ou sem subscritos ou sobrescritos, significarão sequências de sentenças;

c) ' $k$ ', com ou sem subscritos ou sobrescritos, significará um parâmetro;

d) '§', com ou sem subscritos ou sobrescritos, significará uma variável;

e) ' $\alpha(k)^{\prime}$ ' significará a sentença resultante da substituição de todas as ocorrências da variável ' $\S$ ' na fórmula $\alpha$ por ocorrências do pronome ' $k$ ';

d) ' $\alpha\left[k_{1} / / k_{2}\right]$ ' significará a sentença resultante da substituição de uma ou mais ocorrências do pronome $k_{1}$ na sentença $\alpha\left(k_{1}\right)$ por ocorrências do pronome $k_{2}$.

Dispensaremos também os parênteses cuja subtração não produza ambiguidade na leitura das fórmulas. 


\section{Postulados}

Sequente Fundamental:

II (Introdução da Identidade):

IOC (Introdução de Objetos Clássicos): $\alpha \rightarrow \alpha$

$\rightarrow a=a$

$\alpha \rightarrow k=k$

desde que $\alpha$ seja atômica e $k$ ocorra

em $\alpha$.

\section{Esquemas estruturais}

Atenuação (no antecedente): $\quad \frac{\Gamma \rightarrow \Phi}{\alpha, \Gamma \rightarrow \Phi}$

Atenuação (no consequentee): $\quad \frac{\Gamma \rightarrow \Phi}{\Gamma \rightarrow \Phi, \alpha}$

Contração (no antecedente): $\quad \frac{\alpha, \alpha, \Gamma \rightarrow \Phi}{\alpha, \Gamma \rightarrow \Phi}$

Contração (no consequente): $\quad \frac{\Gamma \rightarrow \Phi, \alpha, \alpha}{\Gamma \rightarrow \Phi, \alpha}$

Permutação (no antecedente): $\quad \frac{\Delta, \alpha, \beta, \Gamma \rightarrow \Phi}{\Delta, \beta, \alpha, \Gamma \rightarrow \Phi}$

Permutação (no consequente): $\quad \frac{\Gamma \rightarrow \Phi, \alpha, \beta, \Lambda}{\Gamma \rightarrow \Phi, \beta, \alpha, \Lambda}$

Corte:

$$
\frac{\Gamma \rightarrow \Phi, \alpha \quad \alpha, \Delta \rightarrow \Lambda}{\Gamma, \Delta \rightarrow \Phi, \Lambda}
$$




\section{Esquemas operacionais}

ICA (Introdução da Conjunção no Antecedente): $\frac{\alpha, \Gamma \rightarrow \Phi}{(\alpha \wedge \beta), \Gamma \rightarrow \Phi} \frac{\beta, \Gamma \rightarrow \Phi}{(\alpha \wedge \beta), \Gamma \rightarrow \Phi}$

ICC (Introdução da Conjunção no Consequente): $\frac{\Gamma \rightarrow \Phi, \alpha \quad \Gamma \rightarrow \Phi, \beta}{\Gamma \rightarrow \Phi,(\alpha \wedge \beta)}$

IDA (Introdução da Disjunção no Antecedente): $\frac{\alpha, \Gamma \rightarrow \Phi \quad \beta, \Gamma \rightarrow \Phi}{(\alpha \vee \beta), \Gamma \rightarrow \Phi}$

IDC (Introdução da Disjunção no Consequente): $\frac{\Gamma \rightarrow \Phi, \alpha}{\Gamma \rightarrow \Phi,(\alpha \vee \beta)} \frac{\Gamma \rightarrow \Phi, \beta}{\Gamma \rightarrow \Phi,(\alpha \vee \beta)}$

IUA (Introdução do Universal no Antecedente): $\frac{\alpha(k), \Gamma \rightarrow \Phi \quad \Delta \rightarrow k=k}{\forall \S \alpha, \Gamma, \Delta \rightarrow \Phi}$

IUC (Introdução do Universal no Consequente): $\frac{\Gamma \rightarrow \Phi, \alpha(k)}{\Gamma \rightarrow \Phi, \forall \S \alpha}$

desde que $k$ não ocorra na conclusão.

IEA (Introdução do Existencial no Antecedente): $\frac{\alpha(k), \Gamma \rightarrow \Phi}{\exists \S \alpha, \Gamma \rightarrow \Phi}$

desde que $k$ não ocorra na conclusão.

IEC (Introdução do Existencial no Consequente): $\frac{\Gamma \rightarrow \Phi, \alpha(k)}{\Gamma \rightarrow \Phi, \exists \S \alpha}$

INA (Introdução da Negação no Antecedente): $\frac{\Gamma \rightarrow \Phi, \alpha}{\neg \alpha, \Gamma \rightarrow \Phi}$

INC (Introdução da Negação no Consequente): $\frac{\alpha, \Gamma \rightarrow \Phi}{\Gamma \rightarrow \Phi, \neg \alpha}$ 
IIA (Introdução da Implicação no Antecedente): $\frac{\Gamma \rightarrow \Phi, \alpha \quad \beta, \Delta \rightarrow \Lambda}{(\alpha \supset \beta), \Gamma, \Delta \rightarrow \Phi, \Lambda}$

IIC (Introdução da Implicação no Consequente): $\frac{\alpha, \Gamma \rightarrow \Phi, \beta}{\Gamma \rightarrow \Phi,(\alpha \supset \beta)}$

SI (Substituição de Idênticos):

$$
\frac{\Gamma \rightarrow k_{1}=k_{2} \quad \Delta \rightarrow \alpha}{\Gamma, \Delta \rightarrow \alpha\left[k_{1} / / k_{2}\right]}
$$

\section{Leitura dos sequentes $^{23}$}

Se $\Gamma$ é a sequência de fórmulas $\left\{\alpha_{1}, \ldots, \alpha_{n}\right\}, \Delta$ é a sequência de fórmulas $\left\{\beta_{1}, \ldots, \beta_{k}\right\}$ e $\beta$ é uma fórmula qualquer, então o sequente $\Gamma \rightarrow \Delta$ equivale à seguinte fórmula:
a) $\left(\alpha_{1} \wedge \ldots \wedge \alpha_{n}\right) \supset\left(\beta_{1} \vee \ldots \vee \beta_{k}\right)$, se $\Gamma \neq \emptyset$ e $\Delta \neq \emptyset$;
b) $\beta_{1} \vee \ldots \vee \beta_{k}$, se $\Gamma=\emptyset$ e $\Delta \neq \emptyset$;
c) $\left(\alpha_{1} \wedge \ldots \wedge \alpha_{n}\right) \supset(\beta \wedge \neg \beta)$, se $\Gamma \neq \emptyset$ e $\Delta=\emptyset$;
d) $\beta \wedge \neg \beta$, se $\Gamma=\emptyset$ e $\Delta=\emptyset$.

${ }^{23}$ Cf. GENTZEN, Ibid., pp. 129-130. 


\section{Equivalência entre os sistemas $Q_{G_{1}}$ e $Q_{1}$}

A equivalência entre os sistemas $\mathrm{QG}_{1}$ e $\mathrm{Q}_{1}$ será demonstrada provando-se, por um lado, que toda sentença dedutível em $S$ a partir de um conjunto de sentenças $\Gamma$ é derivável a partir de $\Gamma$ em $\mathrm{QG}_{1}$, e, por outro lado, que toda sentença derivável a partir de um conjunto de sentenças $\Gamma$ em $\mathrm{QG}_{1}$ é dedutível a partir de $\Gamma$ em $\mathrm{Q}_{1}$.

1. Se $\alpha$ é dedutível em $\mathrm{S}$ a partir de um conjunto de sentenças $\Gamma$, então $\alpha$ é derivável a partir de $\Gamma$ em $\mathrm{QG}_{1}$.

Prova: por indução no número $n$ de linhas de uma dedução de $\alpha$ a partir de $\Gamma$ em $\mathrm{S}$.

(i) $\quad n=1$.

Se $n=1$, então $\alpha$ foi deduzida em S a partir de $\Gamma$ por uma das seguintes regras:
a) $\mathrm{P}$;
b) $\mathrm{Ax}_{1}$;
c) $\mathrm{Ax}_{2}$;
d) $\mathrm{T}$. 
a) Se $\alpha$ foi deduzida em $\mathrm{S}$ a partir de $\Gamma$ pela regra $\mathrm{P}$, então $\Gamma=\{\alpha\}$, e, trivialmente, a derivação correspondente de $\alpha$ a partir de $\Gamma$ em $\mathrm{QG}_{1}$ será o sequente fundamental $\alpha \rightarrow \alpha$.

b) Se $\alpha$ foi deduzida em $\mathrm{S}$ a partir de $\Gamma$ pela regra $\mathrm{Ax}_{1}$, então $\Gamma=\emptyset$ e $\alpha$ é $a=a$. Neste caso, portanto, a derivação correspondente de $\alpha$ a partir de $\Gamma$ em $\mathrm{QG}_{1}$ será o postulado $\rightarrow a=a$.

c) Se $\alpha$ foi deduzida em $\mathrm{S}$ a partir de $\Gamma$ pela regra $\mathrm{Ax}_{2}$, então $\Gamma=\emptyset$ e $\alpha$ é $(\beta \supset k=$ $k$ ), onde $\beta$ é atômica e $k$ é um parâmetro que ocorre em $\beta$. Neste caso, portanto, a derivação correspondente de $\alpha$ a partir de $\Gamma$ em $\mathrm{QG}_{1}$ será:

$$
\frac{\beta \rightarrow k=k}{\rightarrow \beta \supset k=k \quad \text { (IIC) }}
$$

d) Se $\alpha$ foi deduzida em $S$ a partir de $\Gamma$ pela regra $T$, então, visto que $n=1, \alpha$ é consequência tautológica de $\emptyset$, e, portanto, $\alpha$ é uma tautologia. Assim, temos a mostrar que todas as tautologias são deriváveis a partir de $\varnothing$ em $\mathrm{QG}_{1}$, para o que mostraremos o seguinte:

I. As tautologias da forma $\alpha \supset(\beta \supset \alpha)$ são deriváveis a partir de $\emptyset$ em $\mathrm{QG}_{1}$;

II. As tautologias da forma $(\alpha \supset \beta) \supset((\alpha \supset(\beta \supset \gamma)) \supset(\alpha \supset \gamma))$ são deriváveis a partir de $\emptyset$ em $\mathrm{QG}_{1}$;

III. As tautologias da forma $(\neg \beta \supset \neg \alpha) \supset(\alpha \supset \beta)$ são deriváveis a partir de $\emptyset$ em $\mathrm{QG}_{1}$;

IV. A regra de Modus Ponens, $\alpha, \alpha \supset \beta / \beta$ é válida em $\mathrm{QG}_{1}$. 
I. $\quad$ Derivação de $\alpha \supset(\beta \supset \alpha)$ a partir de $\emptyset$ em $\mathrm{QG}_{1}$ :

\begin{tabular}{l}
$\frac{\alpha \rightarrow \alpha}{\beta, \alpha \rightarrow \alpha \quad \text { (Atenuação) }}$ \\
\hline$\alpha \rightarrow \beta \supset \alpha \quad$ (IIC) \\
$\rightarrow \alpha \supset(\beta \supset \alpha) \quad$ (IIC)
\end{tabular}

II. $\quad$ Derivação de $(\alpha \supset \beta) \supset((\alpha \supset(\beta \supset \gamma)) \supset(\alpha \supset \gamma))$ a partir de $\emptyset$ em $\mathrm{QG}_{1}$ :

\begin{tabular}{ccr} 
& \multicolumn{1}{c}{$\gamma \rightarrow \gamma$} & \\
\cline { 2 - 2 }$\alpha \supset \beta \rightarrow \alpha \supset \beta$ & $\gamma \rightarrow \alpha \supset \gamma$ & (Atenuação) \\
\hline$(\alpha \supset \beta) \supset \gamma, \alpha \supset \beta \rightarrow \alpha \supset \gamma$ & (IIC) \\
\hline$\alpha \supset \beta \rightarrow((\alpha \supset(\beta \supset \gamma)) \supset(\alpha \supset \gamma))$ & (IIC) \\
\hline$\rightarrow(\alpha \supset \beta) \supset((\alpha \supset(\beta \supset \gamma)) \supset(\alpha \supset \gamma))$ & (IIC)
\end{tabular}

III. Derivação de $(\neg \beta \supset \neg \alpha) \supset(\alpha \supset \beta)$ a partir de $\emptyset$ em $\mathrm{QG}_{1}$ :

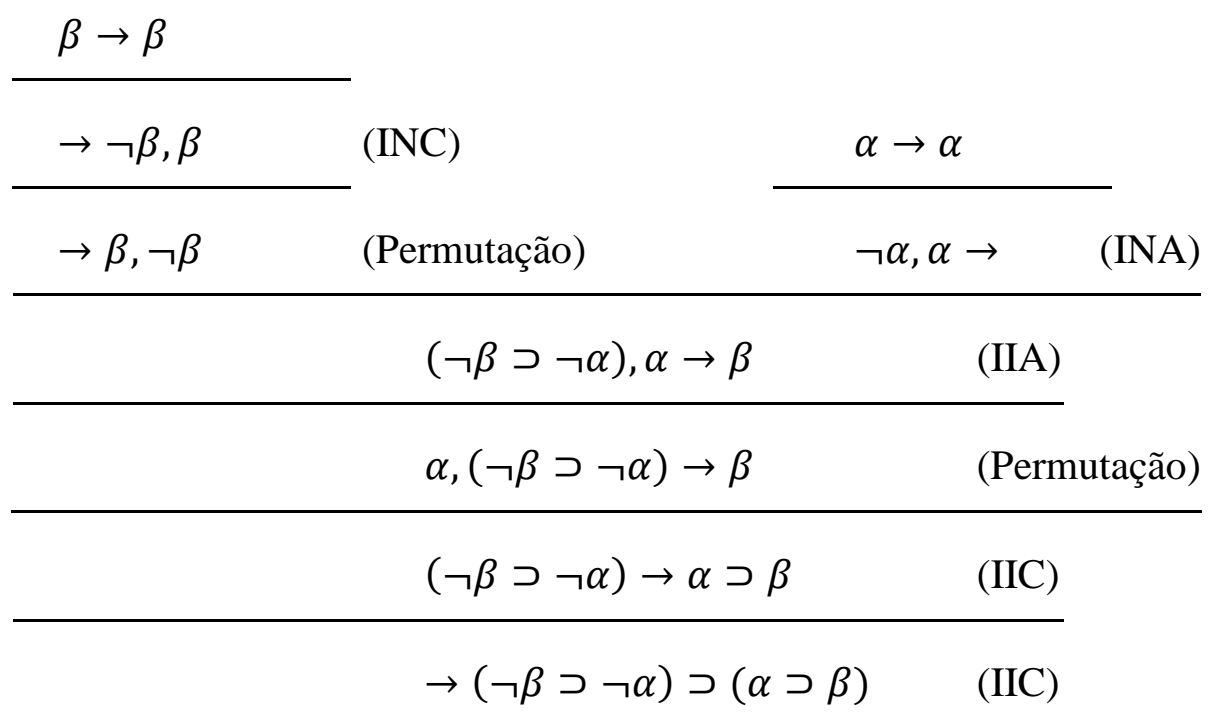


IV. Para mostrar que a regra de Modus Ponens, $\alpha, \alpha \supset \beta / \beta$, é válida em $\mathrm{QG}_{1}$, aceite-se que $\alpha$ e $\alpha \supset \beta$ sejam deriváveis em $\mathrm{QG}_{1}$. Então há derivação, em $\mathrm{QG}_{1}, \quad$ dos sequentes $\rightarrow \alpha$ e $\rightarrow \alpha \supset \beta$. Mas $\rightarrow \alpha \supset \beta$ foi derivado, necessariamente, de $\alpha \rightarrow \beta$ pelo esquema IIC, visto que não há outra forma de ser introduzida a implicação no consequente de um sequente em $\mathrm{QG}_{1}$. Assim, a derivação correspondente de $\beta$ a partir de $\alpha$ e $\alpha \supset \beta$ em $\mathrm{QG}_{1}$ será:

$\frac{\rightarrow \alpha \quad \rightarrow \alpha \supset \beta}{\beta \quad} \quad$ (Corte)

(ii) $\quad n>1$.

Se $n>1$, então $\alpha$ foi deduzida em $\mathrm{S}$ a partir de $\Gamma$ por uma das seguintes regras:
a) $\mathrm{P}$;
b) Ax 1;
c) $\mathrm{Ax} 2$
d) $\mathrm{T}$;
e) $\mathrm{C}$;
f) IU;
g) $\mathrm{GU}$;
h) IE;
i) GE;
j) $\mathrm{E}$;
k) I. 
Suponha-se então que, se $m<n$, e se $\alpha^{\prime}$ foi deduzida em $S$ a partir de $\Gamma^{\prime}$ na linha $m$, então $\alpha^{\prime}$ é derivável a partir de $\Gamma^{\prime}$ em $\mathrm{QG}_{1}$.

a-c) $\operatorname{Se} \alpha$ foi deduzida em $\mathrm{S}$ a partir de $\Gamma$ pela regra $\mathrm{P}, \mathrm{Ax}_{1}$ ou $\mathrm{Ax}_{2}$ em outra linha que não a primeira, então as derivações correspondentes de $\alpha$ a partir de $\Gamma$ em $\mathrm{QG}_{1}$ são, respectivamente, as mesmas apresentadas para quando $n=1$.

d) Se $\alpha$ foi deduzida em $\mathrm{S}$ a partir de $\Gamma$ pela regra $\mathrm{T}$, são duas as possibilidades:

1. $\Gamma=\emptyset$;

2. $\Gamma \neq \varnothing$.

1. Se $\Gamma=\emptyset$, então a derivação correspondente de $\alpha$ a partir de $\Gamma$ em $\mathrm{QG}_{1}$ é a mesma apresentada para quando $n=1$.

2. Se $\Gamma \neq \emptyset$, então $\Gamma=\Gamma_{1} \cup \ldots \cup \Gamma_{l}, \alpha$ é consequência tautológica de $\left\{\alpha_{1}, \ldots, \alpha_{l}\right\}$ e, para $1 \leq i \leq l, \alpha_{i}$ foi deduzida em $\mathrm{S}$ a partir de $\Gamma_{i}$ na linha i. Visto que $l<n$, segue-se pela hipótese da indução que $\alpha_{i}$ é derivável a partir de $\Gamma_{i}$ em $\mathrm{QG}_{1}$. Além disto, se $\alpha$ é consequência tautológica de $\left\{\alpha_{1}, \ldots, \alpha_{l}\right\}$, então $\left(\ldots\left(\left(\alpha_{1} \wedge \alpha_{2}\right) \wedge \ldots\right) \wedge \alpha_{l}\right) \supset \alpha$ é tautologia, e, portanto, $\left(\ldots\left(\left(\alpha_{1} \wedge \alpha_{2}\right) \wedge\right.\right.$ ...) $\left.\wedge \alpha_{l}\right) \supset \alpha$ é derivável a partir de $\emptyset$ em $\mathrm{QG}_{1}$; logo, o sequente $\rightarrow$ $\left(\ldots\left(\left(\alpha_{1} \wedge \alpha_{2}\right) \wedge \ldots\right) \wedge \alpha_{l}\right) \supset \alpha$ é derivável em $\mathrm{QG}_{1}$. Mas $\rightarrow\left(\ldots\left(\left(\alpha_{1} \wedge \alpha_{2}\right) \wedge\right.\right.$ $\left.\ldots) \wedge \alpha_{l}\right) \supset \alpha$ foi derivado, necessariamente, de $\left(\ldots\left(\left(\alpha_{1} \wedge \alpha_{2}\right) \wedge \ldots\right) \wedge\right.$ $\left.\alpha_{l}\right) \rightarrow \alpha$ pelo esquema IIC, visto que não há outra forma de ser introduzida a implicação no consequente de um sequente em $\mathrm{QG}_{1}$. Assim, a derivação correspondente de $\alpha$ a partir de $\Gamma$ em $\mathrm{QG}_{1}$ será: 


$$
\begin{aligned}
& \Gamma_{1} \rightarrow \alpha_{1} \quad \Gamma_{2} \rightarrow \alpha_{2} \\
& \Gamma_{1}, \Gamma_{2} \rightarrow \alpha_{1} \wedge \alpha_{2} \quad(\mathrm{ICC}) \quad \vdots \\
& \Gamma_{l} \rightarrow \alpha_{l} \\
& \Gamma_{1}, \Gamma_{2}, \ldots, \Gamma_{n} \rightarrow\left(\left(\ldots\left(\alpha_{1} \wedge \alpha_{2}\right) \wedge \ldots\right) \wedge \alpha_{l}\right) \quad(\mathrm{ICC}) \quad\left(\ldots\left(\left(\alpha_{1} \wedge \alpha_{2}\right) \wedge \ldots\right) \wedge \alpha_{l}\right) \rightarrow \alpha \\
& \Gamma_{1}, \Gamma_{2}, \ldots, \Gamma_{n} \rightarrow \alpha
\end{aligned}
$$

e) Se $\alpha$ foi deduzida em S a partir de $\Gamma$ pela regra C, então $\alpha$ é $\alpha_{1} \supset \alpha_{2}$, e $\alpha_{2}$ foi deduzida em $S$ a partir de $\Gamma \cup\left\{\alpha_{1}\right\}$ numa linha $l$ tal que $l<n$. Assim, pela hipótese da indução, existe uma derivação em $\mathrm{QG}_{1}$ de $\alpha_{2}$ a partir de $\Gamma \cup\left\{\alpha_{1}\right\}$, e a derivação correspondente de $\alpha$ a partir de $\Gamma$ em $\mathrm{QG}_{1}$ será:

$$
\frac{\Gamma, \alpha_{1} \rightarrow \alpha_{2}}{\Gamma \rightarrow \alpha_{1} \supset \alpha_{2} \text { (IIC) }}
$$

f) Se $\alpha$ foi deduzida em S a partir de $\Gamma$ pela regra IU, então $\alpha$ é $\beta(k), \Gamma=\Gamma_{1} \cup \Gamma_{2}$, $\forall \S \beta$ foi deduzida em $\mathrm{S}$ a partir de $\Gamma_{1}$ numa linha $m$ tal que $m<n$, e $k=k$ foi deduzida em $\mathrm{S}$ a partir de $\Gamma_{2}$ numa linha $m^{\prime}$ tal que $m^{\prime}<n$. Há, portanto, pela hipótese da indução, derivações em $\mathrm{QG}_{1}$ tanto de $\forall \S \beta$ a partir de $\Gamma_{1}$ quanto de $k=k$ a partir de $\Gamma_{2}$. Assim, a derivação correspondente de $\alpha$ a partir de $\Gamma$ em QG1 será:

$$
\begin{array}{ll}
\frac{\beta(k) \rightarrow \beta(k)}{\Gamma_{1} \rightarrow \forall \S \beta} & \Gamma_{2} \rightarrow k=k \\
\hline \Gamma_{1}, \Gamma_{2} \rightarrow \beta(k) & \text { (Corte) }
\end{array}
$$


g) Se $\alpha$ foi deduzida em $S$ a partir de $\Gamma$ pela regra GU, então $\alpha$ é $\forall \S \beta, k$ é um parâmetro que não ocorre em $\beta$ nem em $\Gamma$ e $\beta(k)$ foi deduzida em $S$ a partir de $\Gamma$ numa linha $m<n$. Assim, pela hipótese da indução, há derivação de $\beta(k)$ a partir de $\Gamma$ em $\mathrm{QG}_{1}$, e a derivação correspondente de $\alpha$ a partir de $\Gamma$ em $\mathrm{QG}_{1}$ será:

$$
\frac{\Gamma \rightarrow \beta(k)}{\Gamma \rightarrow \forall \S \beta \quad(\text { IUC) }}
$$

h) Se $\alpha$ foi deduzida em $\mathrm{S}$ a partir de $\Gamma$ pela regra IE, então $\Gamma=\Gamma_{1} \cup \Gamma_{3}, \Gamma_{2}=$ $\{\beta(k)\}, \alpha$ foi deduzida em $\mathrm{S}$ a partir de $\Gamma_{3} \cup \Gamma_{2}$ numa linha $m<n, \exists \S \beta$ foi deduzida em $\mathrm{S}$ a partir de $\Gamma_{1}$ numa linha $l<m$ e $k$ é um parâmetro que não ocorre em $\alpha$, nem em $\beta$, nem em $\Gamma_{3}$. Assim, pela hipótese da indução, existem derivações tanto de $\alpha$ a partir de $\Gamma_{3} \cup\{\beta(k)\}$ quanto de $\exists \S \beta$ a partir de $\Gamma_{1}$ em $\mathrm{QG}_{1}$. Assim, a derivação correspondente de $\alpha$ a partir de $\Gamma$ em $\mathrm{QG}_{1}$ será:

\begin{tabular}{cll} 
& \multicolumn{1}{c}{$\Gamma_{3}, \beta(k) \rightarrow \alpha$} & \\
& $\frac{\beta(k), \Gamma_{3} \rightarrow \alpha}{\text { (Permutação) }}$ \\
\hline$\Gamma_{1} \rightarrow \exists \S \beta$ & $\exists \S \beta, \Gamma_{3} \rightarrow \alpha$ & (IEA) \\
\hline$\Gamma_{1}, \Gamma_{3} \rightarrow \alpha$ & & (Corte)
\end{tabular}

i) Se $\alpha$ foi deduzida em S a partir de $\Gamma$ pela regra GE,então $\alpha$ é $\exists \S \beta$, e $\alpha(k)$ foi deduzida em $\mathrm{S}$ a partir de $\Gamma$ numa linha $m<n$. Assim, pela hipótese da indução, há derivação de $\beta(k)$ a partir de $\Gamma$ em $\mathrm{QG}_{1}$, e a derivação correspondente de $\alpha$ a partir de $\Gamma$ em $\mathrm{QG}_{1}$ será: 


$\frac{\Gamma \rightarrow \beta(k)}{\Gamma \rightarrow \exists \S \beta \quad \text { (IEC) }}$

j) Se $\alpha$ foi deduzida em S a partir de $\Gamma$ pela regra E,então $\alpha$ é $\exists \S \beta$, e $\neg \forall \S \neg \beta$ foi deduzida em S a partir de $\Gamma$ numa linha $m<n$. Assim, pela hipótese da indução, há derivação de $\neg \forall \S \neg \beta$ a partir de $\Gamma$ em $\mathrm{QG}_{1}$, e a derivação correspondente de $\alpha$ a partir de $\Gamma$ em $\mathrm{QG}_{1}$ será:

\begin{tabular}{lll} 
& $\beta(k) \rightarrow \beta(k)$ & \\
& $\rightarrow \beta(k), \neg \beta(k)$ & (INC) \\
& $\rightarrow \neg \beta(k), \beta(k)$ & (Permutação) \\
& $\rightarrow \neg \beta(k), \exists \S \beta$ & (IEC) \\
& $\rightarrow \exists \S \beta, \neg \beta(k)$ & (Permutação) \\
& $\rightarrow \exists \S \beta, \forall \S \neg \beta$ & (IUC) \\
\hline$\Gamma \rightarrow \neg \forall \S \beta \quad \neg \forall \S \neg \beta \rightarrow \exists \S \beta$ & (INA) \\
\hline$\Gamma \rightarrow \exists \S \beta$ & & (Corte)
\end{tabular}

k) Se $\alpha$ foi deduzida em $\mathrm{S}$ a partir de $\Gamma$ pela regra $\mathrm{I}$, então $\Gamma=\Gamma_{1} \cup \Gamma_{2}, \alpha$ é $\beta\left(k_{1} / / k_{2}\right), \mathrm{k}_{1}=\mathrm{k}_{2}$ foi deduzida em $\mathrm{S}$ a partir de $\Gamma_{1}$ numa linha $l<n$ e $\beta$ foi deduzida em $\mathrm{S}$ a partir de $\Gamma_{2}$ numa linha $m<n$. Assim, pela hipótese da indução, há derivações tanto de $\mathrm{k}_{1}=\mathrm{k}_{2}$ a partir de $\Gamma_{1}$ quanto de $\beta$ a partir de $\Gamma_{2}$ em $\mathrm{QG}_{1}$, e a derivação correspondente de $\alpha$ a partir de $\Gamma$ em $\mathrm{QG}_{1}$ será: 


$$
\begin{aligned}
& \begin{array}{ll}
\Gamma_{1} \rightarrow \mathrm{k}_{1}=\mathrm{k}_{2} & \Gamma_{2} \rightarrow \beta \\
\hline
\end{array} \\
& \Gamma_{1}, \Gamma_{2} \rightarrow \beta\left[k_{1} / / k_{2}\right]
\end{aligned}
$$

2. Se $\alpha$ é derivável em $\mathrm{QG}_{1}$ em a partir de um conjunto de sentenças $\Gamma$, então $\alpha$ é dedutível a partir de $\Gamma$ em $\mathrm{S}$.

Prova: por indução no número $n$ de aplicações de esquemas de inferência numa derivação de $\alpha$ a partir de $\Gamma$ em $\mathrm{QG}_{1}$.

(i) $\quad n=0$.

Se $n=0$, então $\alpha$ foi derivada em $\mathrm{QG}_{1}$ a partir de $\Gamma$ por um postulado. Consequentemente, há três possibilidades:

a) $\alpha$ foi derivada em $\mathrm{QG}_{1}$ a partir de $\Gamma$ pelo Sequente Fundamental;

b) $\alpha$ foi derivada em $\mathrm{QG}_{1}$ a partir de $\Gamma$ pela Introdução da Identidade;

c) $\alpha$ foi derivada em $\mathrm{QG}_{1}$ a partir de $\Gamma$ pela Introdução de Objetos Clássicos.

a) Se $\alpha$ foi derivada em $\mathrm{QG}_{1}$ a partir de $\Gamma$ pelo Sequente Fundamental, então $\Gamma=\{\alpha\}$, e a derivação de $\alpha$ a partir de $\Gamma$ em $\mathrm{QG}_{1}$ resume-se ao sequente $\alpha \rightarrow \alpha$. Assim, trivialmente, a dedução correspondente de $\alpha$ a partir de $\Gamma$ em S será:
$\{i\}$
(i) $\alpha$
$(\mathrm{P})$ 
b) Se $\alpha$ foi derivada em $\mathrm{QG}_{1}$ a partir de $\Gamma$ pela Introdução da Identidade, então $\Gamma=\emptyset$, e a derivação de $\alpha$ a partir de $\Gamma$ em $\mathrm{QG}_{1}$ resume-se ao sequente $\rightarrow a=a$. Assim, trivialmente, a dedução correspondente de $\alpha$ a partir de $\Gamma$ em $\mathrm{S}$ será:

$$
\emptyset \quad(i) \quad a=a(\mathrm{Ax} 1)
$$

c) Se $\alpha$ foi derivada em $\mathrm{QG}_{1}$ a partir de $\Gamma$ pela Introdução de Objetos Clássicos, então $\alpha$ é $k=k, \Gamma=\{\beta\}, \beta$ é atômica, $k$ ocorre em $\beta$ e a derivação de $\alpha$ a partir de $\Gamma$ em $\mathrm{QG}_{1}$ resume-se ao sequente $\beta \rightarrow k=k$. Assim, a dedução correspondente de $\alpha$ a partir de $\Gamma$ em S será:

$$
\begin{array}{lll}
\varnothing \quad(i) & \beta \supset k=k & (\mathrm{Ax} 2) \\
\{i+1\}(i+1) & \beta & (\mathrm{P}) \\
\{i+1\}(i+2) & k=k & (i, i+1 / \mathrm{T})
\end{array}
$$

(ii) Se $n>0$, então $\alpha$ foi derivada em $\mathrm{QG}_{1}$ a partir de $\Gamma$ por um dos seguintes esquemas:
a) $\quad \alpha$ foi derivada em $\mathrm{QG}_{1}$ a partir de $\Gamma$ por uma Atenuação no Antecedente;
b) $\alpha$ foi derivada em $\mathrm{QG}_{1}$ a partir de $\Gamma$ por uma Atenuação no Consequente;
c) $\alpha$ foi derivada em $\mathrm{QG}_{1}$ a partir de $\Gamma$ por uma Contração no Antecedente;
d) $\quad \alpha$ foi derivada em $\mathrm{QG}_{1}$ a partir de $\Gamma$ por uma Contração no Consequente;
e) $\alpha$ foi derivada em $\mathrm{QG}_{1}$ a partir de $\Gamma$ por uma Permutação no Antecedente;
f) $\alpha$ foi derivada em $\mathrm{QG}_{1}$ a partir de $\Gamma$ por uma Permutação no Consequente;
g) $\alpha$ foi derivada em $\mathrm{QG}_{1}$ a partir de $\Gamma$ por um Corte; 
h) $\alpha$ foi derivada em $\mathrm{QG}_{1}$ a partir de $\Gamma$ por uma Introdução da Conjunção no Antecedente;

i) $\quad \alpha$ foi derivada em $\mathrm{QG}_{1}$ a partir de $\Gamma$ por uma Introdução da Conjunção no Consequente;

j) $\alpha$ foi derivada em $\mathrm{QG}_{1}$ a partir de $\Gamma$ por uma Introdução da Disjunção no Antecedente;

k) $\alpha$ foi derivada em $\mathrm{QG}_{1}$ a partir de $\Gamma$ por uma Introdução da Disjunção no Consequente;

1) $\alpha$ foi derivada em $\mathrm{QG}_{1}$ a partir de $\Gamma$ por uma Introdução do Universal no Antecedente;

m) $\alpha$ foi derivada em $\mathrm{QG}_{1}$ a partir de $\Gamma$ por uma Introdução do Universal no Consequente;

n) $\alpha$ foi derivada em $\mathrm{QG}_{1}$ a partir de $\Gamma$ por uma Introdução do Existencial no Antecedente;

o) $\alpha$ foi derivada em $\mathrm{QG}_{1}$ a partir de $\Gamma$ por uma Introdução do Existencial no Consequente;

p) $\alpha$ foi derivada em $\mathrm{QG}_{1}$ a partir de $\Gamma$ por uma Introdução da Negação no Antecedente;

q) $\alpha$ foi derivada em $\mathrm{QG}_{1}$ a partir de $\Gamma$ por uma Introdução da Negação no Consequente;

r) $\alpha$ foi derivada em $\mathrm{QG}_{1}$ a partir de $\Gamma$ por uma Introdução da Implicação no Antecedente;

s) $\alpha$ foi derivada em $\mathrm{QG}_{1}$ a partir de $\Gamma$ por uma Introdução da Implicação no Consequente;

t) $\quad \alpha$ foi derivada em $\mathrm{QG}_{1}$ a partir de $\Gamma$ por uma Substituição de Idênticos. 
No que se segue, usaremos a notação $\mu: \Gamma$ para indicar que $\mu$ é o conjunto de números naturais cujos elementos correspondem, na dedução apresentada, aos números das linhas em que as sentenças de $\Gamma$ foram introduzidas pela regra $\mathrm{P}$.

Suponha-se então que, se $m<n$, e se $\alpha^{\prime}$ foi derivada em $\mathrm{QG}_{1}$ a partir de $\Gamma^{\prime}$ mediante um número $m$ de aplicações de esquemas de inferência, então $\alpha^{\prime}$ é dedutível a partir de $\Gamma^{\prime}$ em $\mathrm{S}$.

a) Se $\alpha$ foi derivada em $\mathrm{QG}_{1}$ a partir de $\Gamma$ por uma Atenuação no Antecedente, então $\Gamma=\Delta, \beta$, e há uma derivação em $\mathrm{QG}_{1}$ de $\alpha$ a partir de $\Delta$ mediante um número $m$ de aplicações de esquemas de inferência tal que $m<n$. Logo, pela hipótese da indução, $\alpha$ é dedutível de $\Delta$ em S. Assim, a dedução correspondente de $\alpha$ a partir de $\Gamma$ em $\mathrm{S}$ será, para $\mu: \Delta$ :

$\begin{array}{llll}\mu & (i) & \alpha \\ \mu & (i+1) & \beta \supset \alpha & (i / \mathrm{T}) \\ \{i+2\} & (i+2) & \beta & (\mathrm{P}) \\ \mu \cup\{i+2\} & (i+3) & \alpha & (i+1, i+2 / \mathrm{T})\end{array}$

b) Se $\alpha$ foi derivada em $\mathrm{QG}_{1}$ a partir de $\Gamma$ por uma Atenuação no Consequente, então há uma derivação em $\mathrm{QG}_{1}$ do sequente $\Gamma \rightarrow$ mediante um número $m$ de aplicações de esquemas de inferência tal que $m<n$. Logo, pela hipótese da indução (e pelo significado de $\Gamma \rightarrow$ em $\mathrm{QG}_{1}$ ), $\Gamma \rightarrow \beta \wedge \neg \beta$ é dedutível de $\Gamma$ em S. Assim, a dedução correspondente de $\alpha$ a partir de $\Gamma$ em S será, para $\mu: \Gamma$ :

$\begin{array}{lll}\mu & (i) & \beta \wedge \neg \beta \\ \mu & (i+1) & \alpha\end{array}$


c) Se $\alpha$ foi derivada em $\mathrm{QG}_{1}$ a partir de $\Gamma$ por uma Contração no Antecedente, então $\Gamma=\beta, \Delta$, e há uma derivação em $\mathrm{QG}_{1}$ de $\alpha$ a partir de $\beta, \beta, \Delta$ mediante um número $m$ de aplicações de esquemas de inferência tal que $m<n$. Logo, pela hipótese da indução, $\alpha$ é dedutível do conjunto dos termos da sequência $\beta, \beta, \Delta$ em S. Mas o conjunto dos termos da sequência $\beta, \beta, \Delta$ é idêntico ao conjunto dos termos da sequência $\beta, \Delta$; assim, há uma dedução correspondente de $\alpha$ a partir de $\Gamma$ em S.

d) Se $\alpha$ foi derivada em $\mathrm{QG}_{1}$ a partir de $\Gamma$ por uma Contração no Consequente, então há uma derivação em $\mathrm{QG}_{1}$ de $\alpha, \alpha$ a partir de $\Gamma$ mediante um número $m$ de aplicações de esquemas de inferência tal que $m<n$. Logo, pela hipótese da indução (e pelo significado de $\alpha, \alpha$ no consequente de um sequente em $\mathrm{QG}_{1}$ ), $\alpha \vee \alpha$ é dedutível de $\Gamma$ em $\mathrm{S}$. Assim, a dedução correspondente de $\alpha$ a partir de $\Gamma$ em S será, para $\mu: \Gamma$ :

$\begin{array}{lll}\mu & (i) & \alpha \vee \alpha \\ \mu & (i+1) & \alpha\end{array}$

e) Se $\alpha$ foi derivada em $\mathrm{QG}_{1}$ a partir de $\Gamma$ por uma Permutação no Antecedente, então $\Gamma=\Delta_{1}, \beta_{1}, \beta_{2}, \Delta_{2}$, e há uma derivação em $\mathrm{QG}_{1}$ de $\alpha$ a partir de $\Delta_{1}, \beta_{2}, \beta_{1}, \Delta_{2}$ mediante um número $m$ de aplicações de esquemas de inferência tal que $m<n$. Logo, pela hipótese da indução, $\alpha$ é dedutível do conjunto dos termos da sequência $\Delta_{1}, \beta_{2}, \beta_{1}, \Delta_{2}$ em $\mathrm{S}$. Mas o conjunto dos termos da 
sequência $\Delta_{1}, \beta_{2}, \beta_{1}, \Delta_{2}$ é idêntico ao conjunto dos termos da sequência $\Delta_{1}, \beta_{1}, \beta_{2}, \Delta_{2}$; assim, há uma dedução correspondente de $\alpha$ a partir de $\Gamma$ em $\mathrm{S}$.

f) Se $\alpha$ foi derivada em $\mathrm{QG}_{1}$ a partir de $\Gamma$ por uma Permutação no Consequente, então $\alpha$ é $\beta_{1}, \beta_{2}$, e há uma derivação em $\mathrm{QG}_{1}$ de $\beta_{2}, \beta_{1}$ a partir de $\Gamma$ mediante um número $m$ de aplicações de esquemas de inferência tal que $m<n$. Logo, pela hipótese da indução (e pelo significado de $\beta_{2}, \beta_{1}$ no consequente de um sequente em $\left.\mathrm{QG}_{1}\right), \beta_{2} \vee \beta_{1}$ é dedutível de $\Gamma$ em $\mathrm{S}$. Assim, a dedução correspondente de $\alpha$ a partir de $\Gamma$ em $\mathrm{S}$ será, para $\mu: \Gamma$ :

$$
\begin{array}{llll}
\mu & (i) & \beta_{2} \vee \beta_{1} & \\
\mu & (i+1) & \beta_{1} \vee \beta_{2} & (i+1 / \mathrm{T})
\end{array}
$$

g) Se $\alpha$ foi derivada em $\mathrm{QG}_{1}$ a partir de $\Gamma$ por um Corte, então $\Gamma=\Delta_{1}$, $\Delta_{2}$, e há $\beta$ tal que, para $l<n, m<n$ :

1. $\Delta_{1} \rightarrow \alpha, \beta$ foi derivado em $\mathrm{QG}_{1}$ mediante um número $l$ de aplicações de esquemas de inferência e $\beta, \Delta_{2} \rightarrow$ foi derivado em $\mathrm{QG}_{1}$ mediante um número $m$ de aplicações de esquemas de inferência;

2. $\Delta_{1} \rightarrow \beta$ foi derivado em $\mathrm{QG}_{1}$ mediante um número $l$ de aplicações de esquemas de inferência e $\beta, \Delta_{2} \rightarrow \alpha$ foi derivado em $\mathrm{QG}_{1}$ mediante um número $m$ de aplicações de esquemas de inferência. 
1. Se $\Delta_{1} \rightarrow \alpha, \beta$ foi derivado em $\mathrm{QG}_{1}$ mediante um número $l$ de aplicações de esquemas de inferência, então, pela hipótese da indução (e pelo significado de $\alpha, \beta$ no consequente de um sequente em $\left.\mathrm{QG}_{1}\right), \alpha \vee \beta$ é dedutível a partir de $\Delta_{1}$ em $\mathrm{S}$. Além disso, se $\beta, \Delta_{2} \rightarrow$ foi derivado em $\mathrm{QG}_{1}$ mediante um número $m$ de aplicações de esquemas de inferência, então, pela hipótese da indução (e pelo significado de $\beta, \Delta_{2} \rightarrow$ em $\mathrm{QG}_{1}$ ), $\beta^{\prime} \wedge \neg \beta^{\prime}$ é dedutível de $\beta, \Delta_{2}$ em S. Assim, a dedução correspondente de $\alpha$ a partir de $\Gamma$ em $\mathrm{S}$ será, para $\mu_{1}: \Delta_{1}, \mu_{2}: \Delta_{2}:$
$\mu_{1}$
(i)
$\alpha \vee \beta$
$\left\{i^{\prime}\right\}$
$\left(i^{\prime}\right)$
$\beta$
$(\mathrm{P})$
$\vdots$
$\mu_{2} \cup\left\{i^{\prime}\right\}$
$\left(i^{\prime \prime}\right)$
$\beta^{\prime} \wedge \neg \beta^{\prime}$
$\mu_{2}$
$\left(i^{\prime \prime}+1\right)$
$\beta \supset\left(\beta^{\prime} \wedge \neg \beta^{\prime}\right)$
$\left(i^{\prime \prime}, i^{\prime \prime}+1 / \mathrm{C}\right)$
$\mu_{1} \cup \mu_{2}$
$\left(i^{\prime \prime}+2\right)$
$\alpha$
$\left(i, i^{\prime \prime}+1 / \mathrm{T}\right)$

2. Se $\Delta_{1} \rightarrow \beta$ foi derivado em $\mathrm{QG}_{1}$ mediante um número $l$ de aplicações de esquemas de inferência, então, pela hipótese da indução, $\beta$ é dedutível a partir de $\Delta_{1}$ em $\mathrm{S}$. Além disto, se $\beta, \Delta_{2} \rightarrow \alpha$ foi derivado em $\mathrm{QG}_{1}$ mediante um número $m$ de aplicações de esquemas de inferência, então $\alpha$ é dedutível a partir de $\{\beta\} \cup \Delta_{2}$ em S. Assim, a dedução correspondente de $\alpha$ a partir de $\Gamma$ em S será, para $\mu_{1}: \Delta_{1}, \mu_{2}: \Delta_{2}$ : 


\begin{tabular}{|c|c|c|c|}
\hline \multirow[t]{2}{*}{$\mu_{1}$} & (i) & $\beta$ & \\
\hline & $\vdots$ & & \\
\hline \multirow[t]{2}{*}{$i^{\prime}$} & $\left(i^{\prime}\right)$ & $\beta$ & \\
\hline & $\vdots$ & & \\
\hline$\mu_{2} \cup\left\{i^{\prime}\right\}$ & $\left(i^{\prime \prime}\right)$ & $\alpha$ & \\
\hline$\mu_{2}$ & $\left(i^{\prime \prime}+1\right)$ & $\beta \supset \alpha$ & $\left(i^{\prime}, i^{\prime \prime} / \mathrm{C}\right)$ \\
\hline$\mu_{1} \cup \mu_{2}$ & $\left(i^{\prime \prime}+1\right)$ & $\alpha$ & $\left(i, i^{\prime \prime}+1 / \mathrm{T}\right)$ \\
\hline
\end{tabular}

h) Se $\alpha$ foi derivada em $\mathrm{QG}_{1}$ a partir de $\Gamma$ por uma Introdução da Conjunção no Antecedente, então $\Gamma=\beta_{1} \wedge \beta_{2}$, $\Delta$, e há derivação de $\alpha$ em $\mathrm{QG}_{1}$ a partir de $\beta_{1}, \Delta$ mediante um número $l$ (tal que $l<n$ ) de aplicações de esquemas de inferência ou há derivação de $\alpha$ em $\mathrm{QG}_{1}$ a partir de $\beta_{2}, \Delta$ mediante um número $m$ (tal que $m<n)$ de aplicações de esquemas de inferência. Logo, pela hipótese da indução, $\alpha$ é dedutível a partir de $\beta_{1}, \Delta$ ou a partir de $\beta_{2}, \Delta$ em S. Assim, a deduçao correspondente de $\alpha$ a partir de $\Gamma$ em S será, para $1 \leq j \leq 2, \mu$ : $\Delta$ :
$\{i\}$
(i)
$\beta_{j}$
$(\mathrm{P})$
$\mu \cup\{i\}$
$\left(i^{\prime}\right)$
$\alpha$
$\mu$
$\left(i^{\prime}+1\right)$
$\beta_{j} \supset \alpha$
$\left(i, i^{\prime} / \mathrm{C}\right)$
$\left\{i^{\prime}+2\right\} \quad\left(i^{\prime}+2\right) \quad \beta_{1} \wedge \beta_{2}$
$(\mathrm{P})$
$\mu \cup\left\{i^{\prime}+2\right\} \quad\left(i^{\prime}+3\right)$
$\alpha$
$\left(i^{\prime}+1, i^{\prime}+2 / \mathrm{T}\right)$ 
i) Se $\alpha$ foi derivada em $\mathrm{QG}_{1}$ a partir de $\Gamma$ por uma Introdução da Conjunção no Consequente, então $\alpha$ é $\beta_{1} \wedge \beta_{2}$, e há derivações em $\mathrm{QG}_{1}$ tanto de $\beta_{1}$ a partir de $\Gamma$ mediante um número $l$ (tal que $l<n$ ) de aplicações de esquemas de inferência quanto de $\beta_{2}$ a partir de $\Gamma$ mediante um número $m$ (tal que $m<n$ ) de aplicações de esquemas de inferência. Logo, pela hipótese da indução, tanto $\beta_{1}$ é dedutível a partir de $\Gamma$ em $\mathrm{S}$ quanto $\beta_{2}$ é dedutível a partir de $\Gamma$ em $\mathrm{S}$. Assim, a deduçao correspondente de $\alpha$ a partir de $\Gamma$ em S será, para $\mu: \Gamma$ :

$\begin{array}{llll}\mu & (i) & \beta_{1} & \\ & \vdots & \\ \mu & \left(i^{\prime}\right) & \beta_{2} & \\ \mu & \left(i^{\prime}+1\right) & \beta_{1} \wedge \beta_{2} \quad\left(i, i^{\prime} / \mathrm{T}\right)\end{array}$

j) Se $\alpha$ foi derivada em $\mathrm{QG}_{1}$ a partir de $\Gamma$ por uma Introdução da Disjunção no Antecedente, então $\Gamma=\beta_{1} \vee \beta_{2}, \Delta$, e há derivações de $\alpha$ em $\mathrm{QG}_{1}$ tanto a partir de $\beta_{1}, \Delta$ mediante um número $l$ (tal que $l<n$ ) de aplicações de esquemas de inferência quanto a partir de $\beta_{2}, \Delta$ mediante um número $m$ (tal que $m<n$ ) de aplicações de esquemas de inferência. Logo, pela hipótese da indução, $\alpha$ é dedutível tanto a partir de $\beta_{1}, \Delta$ quanto a partir de $\beta_{2}, \Delta$ em S. Assim, a deduçao correspondente de $\alpha$ a partir de $\Gamma$ em S será, para $\mu: \Delta$ :

$\{i\}$

$\left\{i^{\prime}\right\}$ (i)

$\vdots$

$\left(i^{\prime}\right)$ $\beta_{1}$

$\beta_{2}$ 


$\begin{array}{llll}\mu \cup\{i\} & (j) & \alpha \\ & \vdots & \\ \mu \cup\left\{i^{\prime}\right\} & \left(j^{\prime}\right) & \alpha & \\ \mu & \left(j^{\prime}+1\right) & \beta_{1} \supset \alpha & (i, j / \mathrm{C}) \\ \mu & \left(j^{\prime}+2\right) & \beta_{2} \supset \alpha & \left(i^{\prime}, j^{\prime} / \mathrm{C}\right) \\ \left\{j^{\prime}+3\right\} & \left(j^{\prime}+3\right) & \beta_{1} \vee \beta_{2} & (\mathrm{P}) \\ \mu \cup\left\{j^{\prime}+3\right\} & \left(i^{\prime}+4\right) & \alpha & \left(j^{\prime}+1, j^{\prime}+2, j^{\prime}+3 / \mathrm{T}\right)\end{array}$

k) Se $\alpha$ foi derivada em $\mathrm{QG}_{1}$ a partir de $\Gamma$ por uma Introdução da Disjunção no Consequente, então $\alpha$ é $\beta_{1} \vee \beta_{2}$, e há derivação de $\beta_{1}$ em $\mathrm{QG}_{1}$ a partir de $\Gamma$ mediante um número $l($ tal que $l<n)$ de aplicações de esquemas de inferência ou há derivação de $\beta_{2}$ em $\mathrm{QG}_{1}$ a partir de $\Gamma$ mediante um número $m$ (tal que $m<n)$ de aplicações de esquemas de inferência. Logo, pela hipótese da indução, $\beta_{1}$ é dedutível a partir de $\Gamma$ em $\mathrm{S}$ ou $\beta_{2}$ é dedutível a partir de $\Gamma$ em $\mathrm{S}$. Assim, a deduçao correspondente de $\alpha$ a partir de $\Gamma$ em S será, para $1 \leq j \leq 2$, $\mu: \Gamma:$

$\begin{array}{lll}\mu & (i) & \beta_{j} \\ \mu & (i+1) & \beta_{1} \vee \beta_{2}\end{array}$

1) Se $\alpha$ foi derivada em $\mathrm{QG}_{1}$ a partir de $\Gamma$ por uma Introdução do Universal no Antecedente, então $\Gamma=\forall \S \beta, \Delta$, e há derivações em $\mathrm{QG}_{1}$ tanto de $\alpha$ a partir de $\beta(k), \Delta$ mediante um número $l$ (tal que $l<n$ ) de aplicações de esquemas de inferência quanto de $k=k$ a partir de $\emptyset$ mediante um número $m$ (tal que $m<$ 
n) de aplicações de esquemas de inferência. Logo, pela hipótese da indução, tanto $\alpha$ é dedutível a partir de $\beta(k), \Delta$ em S quanto $k=k$ é dedutível a partir de $\emptyset$ em S. Assim, a deduçao correspondente de $\alpha$ a partir de $\Gamma$ em S será, para $\mu: \Delta:$

$\begin{array}{llll}\{i\} & (i) & \beta(k) \\ & \vdots & \\ \mu \cup\{i\} & \left(i^{\prime}\right) & \alpha \\ & \vdots & k=k & (\mathrm{P}) \\ \varnothing & (j) & \forall \S \beta & (j, j+1 / \mathrm{IU}) \\ \{j+1\} & (j+1) & \beta(k) & \left(i, i^{\prime} / \mathrm{C}\right) \\ \{j+1\} & (j+2) & \beta(k) \supset \alpha & (j+2, j+3 / \mathrm{T}) \\ \mu & (j+3) & \alpha\end{array}$

m) Se $\alpha$ foi derivada em $\mathrm{QG}_{1}$ a partir de $\Gamma$ por uma Introdução do Universal no Consequente, então $\alpha$ é $\forall \S \beta, k$ não ocorre em $\Gamma$ nem em $\beta$ e há derivação em $\mathrm{QG}_{1}$ de $\beta(k)$ a partir de $\Gamma$ mediante um número $m$ (tal que $m<n$ ) de aplicações de esquemas de inferência. Logo, pela hipótese da indução, $\beta(k)$ é dedutível a partir de $\Gamma$ em $\mathrm{S}$. Assim, a deduçao correspondente de $\alpha$ a partir de $\Gamma$ em $\mathrm{S}$ será, $\operatorname{para} \mu: \Gamma$ :

$\begin{array}{llll}\mu & (i) & \beta(k) & \\ \mu & (i+1) & \forall \S \beta & (i / \mathrm{GU})\end{array}$


n) Se $\alpha$ foi derivada em $\mathrm{QG}_{1}$ a partir de $\Gamma$ por uma Introdução do Existencial no Antecedente, então $\Gamma=\exists \S \beta, \Delta, k$ não ocorre em $\Gamma$ nem em $\beta$ nem em $\alpha$, e há derivação em $\mathrm{QG}_{1}$ de $\alpha$ a partir de $\beta(k), \Delta$ mediante um número $m$ (tal que $m<n)$ de aplicações de esquemas de inferência. Logo, pela hipótese da indução, $\alpha$ é dedutível a partir de $\{\beta(k)\} \cup \Delta$ em S. Assim, a deduçao correspondente de $\alpha$ a partir de $\Gamma$ em S será, para $\mu: \Delta$ :

$\begin{array}{lll}\{i\} & (i) & \beta(k) \\ & \vdots & \\ \mu \cup\{i\} & (j) & \alpha \\ \{j+1\} & (j+1) & \exists \S \beta \\ \mu \cup\{j+1\} & (j+2) & \alpha\end{array}$

o) Se $\alpha$ foi derivada em $\mathrm{QG}_{1}$ a partir de $\Gamma$ por uma Introdução do Existencial no Consequente, então $\alpha$ é $\exists \S \beta$, e há derivação em $\mathrm{QG}_{1}$ de $\beta(k)$ a partir de $\Gamma$ mediante um número $m$ (tal que $m<n$ ) de aplicações de esquemas de inferência. Logo, pela hipótese da indução, $\beta(k)$ é dedutível a partir de $\Gamma$ em $\mathrm{S}$. Assim, a deduçao correspondente de $\alpha$ a partir de $\Gamma$ em S será, para $\mu: \Gamma$ :

$\begin{array}{llll}\mu & (i) & \beta(k) & \\ \mu & (i+1) & \exists \S \beta & (i / \mathrm{GE})\end{array}$

p) Se $\alpha$ foi derivada em $\mathrm{QG}_{1}$ a partir de $\Gamma$ por uma Introdução da Negação no Antecedente, então $\Gamma=\neg \beta, \Delta$, e há uma derivação em $\mathrm{QG}_{1}$ de $\alpha, \beta$ a partir de $\Delta$ mediante um número $m$ de aplicações de esquemas de inferência tal que $m<n$. 
Logo, pela hipótese da indução (e pela interpretação de $\alpha, \beta$ no consequente de um sequente em $\left.\mathrm{QG}_{1}\right), \alpha \vee \beta$ é dedutível a partir de $\Delta$ em $\mathrm{S}$. Assim, a dedução correspondente de $\alpha$ a partir de $\Gamma$ em S será, para $\mu: \Delta$ :

$\begin{array}{llll}\mu & (i) & \alpha \vee \beta & \\ \{i+1\} & (i+1) & \neg \beta & (\mathrm{P}) \\ \mu \cup\{i+1\} & (i+2) & \alpha & (i, i+1 / \mathrm{T})\end{array}$

q) Se $\alpha$ foi derivada em $\mathrm{QG}_{1}$ a partir de $\Gamma$ por uma Introdução da Negação no Consequente, então $\alpha$ é $\neg \beta$, e há uma derivação em $\mathrm{QG}_{1}$ do sequente $\beta, \Gamma \rightarrow$ mediante um número $m$ de aplicações de esquemas de inferência tal que $m<n$. Logo, pela hipótese da indução (e pelo significado de $\beta, \Gamma \rightarrow$ em $\mathrm{QG}_{1}$ ), $\beta^{\prime} \wedge \neg \beta^{\prime}$ é dedutível a partir de $\{\beta\} \cup \Gamma$ em S. Assim, a dedução correspondente de $\alpha$ a partir de $\Gamma$ em S será, para $\mu: \Gamma$ :
$\{i\}$
(i)
$\beta$
$\mu \cup\{i\}$
$\left(i^{\prime}\right)$
$\beta^{\prime} \wedge \neg \beta^{\prime}$
$\mu$
$\left(i^{\prime}+1\right)$
$\beta \supset\left(\beta^{\prime} \wedge \neg \beta^{\prime}\right)$
$\left(i, i^{\prime} / \mathrm{C}\right)$
$\mu$
$\left(i^{\prime}+2\right) \quad \neg \beta$
$\left(i^{\prime}+1 / \mathrm{T}\right)$

r) Se $\alpha$ foi derivada em $\mathrm{QG}_{1}$ a partir de $\Gamma$ por uma Introdução da Implicação no Antecedente, então $\Gamma=\left\{\beta_{1} \supset \beta_{2}\right\} \cup \Delta_{1} \cup \Delta_{2}$, e, para $l<n, m<n$ : 
1. $\Delta_{1} \rightarrow \alpha, \beta_{1}$ foi derivado em $\mathrm{QG}_{1}$ mediante um número $l$ de aplicações de esquemas de inferência e $\beta_{2}, \Delta_{2} \rightarrow$ foi derivado em $\mathrm{QG}_{1}$ mediante um número $m$ de aplicações de esquemas de inferência;

2. $\Delta_{1} \rightarrow \beta_{1}$ foi derivado em $\mathrm{QG}_{1}$ mediante um número $l$ de aplicações de esquemas de inferência e $\beta_{2}, \Delta_{2} \rightarrow \alpha$ foi derivado em $\mathrm{QG}_{1}$ mediante um número $m$ de aplicações de esquemas de inferência.

1. Se $\Delta_{1} \rightarrow \alpha, \beta_{1}$ foi derivado em $\mathrm{QG}_{1}$ mediante um número $l$ de aplicações de esquemas de inferência, então, pela hipótese da indução, (e pelo significado de $\alpha, \beta_{1}$ no consequente de um sequente em $\left.\mathrm{QG}_{1}\right), \alpha \vee \beta_{1}$ é dedutível a partir de $\Delta_{1}$ em $\mathrm{S}$. Além disso, se $\beta_{2}, \Delta_{2} \rightarrow$ foi derivado em $\mathrm{QG}_{1}$ mediante um número $m$ de aplicações de esquemas de inferência, então, pela hipótese da indução (e pelo significado de $\beta_{2}, \Delta_{2} \rightarrow$ em $\mathrm{QG}_{1}$ ), $\beta^{\prime} \wedge \neg \beta^{\prime}$ é dedutível a partir de $\left\{\beta_{2}\right\} \cup \Delta_{2}$ em S. Assim, a dedução correspondente de $\alpha$ a partir de $\Gamma$ em S será, para $\mu_{1}: \Delta_{1}, \mu_{2}: \Delta_{2}$ :
$\mu_{1}$
(i)
$\alpha \vee \beta_{1}$
$\left\{i^{\prime}\right\}$
$\left(i^{\prime}\right)$
$\beta_{2}$
$\mu_{2} \cup\left\{i^{\prime}\right\}$
$\left(i^{\prime \prime}\right)$
$\beta^{\prime} \wedge \neg \beta^{\prime}$
$\mu_{2}$
$\left(i^{\prime \prime}+1\right) \quad \beta_{2} \supset\left(\beta^{\prime} \wedge \neg \beta^{\prime}\right) \quad\left(i^{\prime \prime}, i^{\prime \prime}+1 / \mathrm{C}\right)$
$\left\{i^{\prime \prime}+2\right\}$
$\left(i^{\prime \prime}+2\right) \quad \beta_{1} \supset \beta_{2}$
(P)
$\mu_{1} \cup \mu_{2} \cup\left\{i^{\prime \prime}+2\right\} \quad\left(i^{\prime \prime}+3\right) \quad \alpha$
$\left(i, i^{\prime \prime}+1, i^{\prime \prime}+2 / \mathrm{T}\right)$ 
2. Se $\Delta_{1} \rightarrow \beta_{1}$ foi derivado em $\mathrm{QG}_{1}$ mediante um número $l$ de aplicações de esquemas de inferência, então, pela hipótese da indução, $\beta_{1}$ é dedutível a partir de $\Delta_{1}$ em $\mathrm{S}$. Além disso, se $\beta_{2}, \Delta_{2} \rightarrow \alpha$ foi derivado em $\mathrm{QG}_{1}$ mediante um número $m$ de aplicações de esquemas de inferência, então, pela hipótese da indução, $\alpha$ é dedutível a partir de $\left\{\beta_{2}\right\} \cup \Delta_{2}$ em S. Assim, a dedução correspondente de $\alpha$ a partir de $\Gamma$ em $S$ será, para $\mu_{1}: \Delta_{1}, \mu_{2}: \Delta_{2}$ :

$\begin{array}{llll}\mu_{1} & (i) & \beta_{1} & \\ & \vdots & & \\ & & & \\ \left\{i^{\prime}\right\} & \left(i^{\prime}\right) & \beta_{2} & (\mathrm{P}) \\ & \vdots & & \\ \left\{i^{\prime}\right\} \cup \mu_{2} & \left(i^{\prime \prime}\right) & \alpha & \left(i^{\prime}, i^{\prime \prime} / \mathrm{C}\right) \\ \mu_{2} & \left(i^{\prime \prime}+1\right) & \beta_{2} \supset \alpha & (\mathrm{P}) \\ \left\{\mathrm{i}^{\prime \prime}+2\right\} & \left(i^{\prime \prime}+2\right) & \beta_{1} \supset \beta_{2} & \left(i, i^{\prime \prime}+1, i^{\prime \prime}+2 / \mathrm{T}\right) \\ \mu_{1} \cup \mu_{2} \cup\left\{\mathrm{i}^{\prime \prime}+2\right\} & \left(i^{\prime \prime}+3\right) & \alpha & \end{array}$

s) Se $\alpha$ foi derivada em $\mathrm{QG}_{1}$ a partir de $\Gamma$ por uma Introdução da Implicação no Consequente, então $\alpha$ é $\beta_{1} \supset \beta_{2}$, e há uma derivação em $\mathrm{QG}_{1}$ de $\beta_{2}$ a partir de $\beta_{1}, \Gamma$ mediante um número $m$ de aplicações de esquemas de inferência tal que $m<n$. Logo, pela hipótese da indução, $\beta_{2}$ é dedutível a partir de $\left\{\beta_{1}\right\} \cup \Gamma$ em $\mathrm{S}$. Assim, a dedução correspondente de $\alpha$ a partir de $\Gamma$ em S será, para $\mu: \Gamma$ : 


$\begin{array}{llll}\{i\} & (i) & \beta_{1} & (\mathrm{P}) \\ & \vdots & & \\ \mu \cup\{i\} & \left(i^{\prime}\right) & \beta_{2} & \\ \mu & \left(i^{\prime}+1\right) & \beta_{1} \supset \beta_{2} & \left(i, i^{\prime} / \mathrm{C}\right)\end{array}$

t) Se $\alpha$ foi derivada em $\mathrm{QG}_{1}$ a partir de $\Gamma$ por uma Substituição de Idênticos, então $\Gamma=\Delta_{1} \cup \Delta_{2}$, existe $\beta$ tal que $\alpha$ é $\beta\left[k_{1} / / k_{2}\right]$ e há derivações em $\mathrm{QG}_{1}$ tanto de $k_{1}=k_{2}$ a partir de $\Delta_{1}$ mediante um número $l$ (tal que $l<n$ ) de aplicações de esquemas de inferência quanto de $\beta$ a partir de $\Delta_{2}$ mediante um número $m$ (tal que $m<n$ ) de aplicações de esquemas de inferência. Logo, pela hipótese da indução, tanto $k_{1}=k_{2}$ é dedutível a partir de $\Delta_{1}$ em $\mathrm{S}$ quanto $\beta$ é dedutível a partir de $\Delta_{2}$ em S. Assim, a dedução correspondente de $\alpha$ a partir de $\Gamma$ em S será, para $\mu_{1}: \Delta_{1}, \mu_{2}: \Delta_{2}$
$\mu_{1}$
(i)
$k_{1}=k_{2}$
$(\mathrm{P})$
$\vdots$
$\mu_{2}$
$\left(i^{\prime}\right)$
$\beta$
$\mu_{1} \cup \mu_{2}$
$\left(i^{\prime}+1\right)$
$\beta\left[k_{1} / / k_{2}\right]$
$\left(i, i^{\prime} / \mathrm{I}\right)$ 


\section{Conclusão}

O sistema $\mathrm{Q}_{1 \mathrm{p}}$, conforme assinalado, é uma extensão conservativa do sistema $\mathrm{Q}_{1}$. Consequentemente, se uma sentença for válida em $\mathrm{Q}_{1}$, essa sentença também será válida em $\mathrm{Q}_{1 \mathrm{p}}$. Assim, qualquer sentença válida em $\mathrm{Q}_{1}$ será teorema de $\mathrm{S}$, visto que $\mathrm{S}$ é completo relativamente a $\mathrm{Q}_{1 \mathrm{p}}$, e, portanto, $\mathrm{S}$ é completo relativamente a $\mathrm{Q}_{1}$. Por outro lado, como Adorno mostrou ${ }^{24}$, todo teorema de $\mathrm{S}$ que é uma fórmula da linguagem de $\mathrm{Q}_{1}$ é válido em $\mathrm{Q}_{1}$. Logo, uma vez demonstrada a equivalência entre os sistemas $\mathrm{QG}_{1} \mathrm{e}$ $\mathrm{S}$, segue-se dessa equivalência que o sistema $\mathrm{QG}_{1}$ é também correto e completo relativamente a $\mathrm{Q}_{1}$.

Além disso, visto que o sistema $\mathrm{Q}_{2}$ é tradutível em $\mathrm{Q}_{1}$, segue-se dessas mesmas razões que um teorema de correção e completude do sistema $\mathrm{QG}_{1}$ com respeito a $\mathrm{Q}_{2}$ pode ser formulado nos seguintes termos: seja $\alpha$ uma fórmula de $\mathrm{Q}_{2}$ e $\alpha^{*}$ uma tradução de $\alpha$ em $\mathrm{Q}_{1}$. Então $\alpha$ é válida em $\mathrm{Q}_{2}$ se e somente se $\alpha^{*}$ é demonstrável em $\mathrm{S}$ - portanto, em $\mathrm{QG}_{1}$.

\footnotetext{
${ }^{24}$ Cf. Adorno, Fernando Paulo Christe. Dois Sistemas de Lógica Não Reflexiva. Dissertação de Mestrado. São Paulo: Universidade de São Paulo, 2001.
} 


\section{Bibliografia}

Adorno, Fernando Paulo Christe. Dois Sistemas de Lógica Não Reflexiva.

Dissertação de Mestrado. São Paulo: Universidade de São Paulo, 2001.

ChOMsky, Noam. Language and Mind. New York: Harcourt, Brace \& World, 1968.

GENTZEN, Gerhard. Recherches sur La Déduction Logique. Trad. Robert Feys e Jean Ladrière. Paris: Presses Universitaires de France, 1955.

LOPARIĆ, Andréa. Les Négations et Les Univers du Discours. In: Lacan avec Les Philosophes. Paris: Albin Michel, 1991.

MAtes, Benson. Lógica Elementar. Trad. Leônidas H. B. Hegenberg e Octanny Silveira Mota. São Paulo: Companhia Editora Nacional, 1968.

RoudinESCO, Elisabeth. Jacques Lacan: Esquisse d'Une Vie, Histoire d'Un Système de Pensée. Paris: Librairie Arthème Fayard, 1993.

Smullyan, Raymond M. First-Order Logic. New York: Dover, 1968. 